\title{
Regioselective and stereospecific hydroxylation of GR24 by Sorghum bicolor and evaluation of germination inducing activities of hydroxylated GR24 stereoisomers toward seeds of Striga species
}

Kotomi Ueno ${ }^{\dagger}$, Shunsuke Ishiwa, Hitomi Nakashima, Masaharu Mizutani, Hirosato Takikawa, Yukihiro Sugimoto*

Graduate School of Agricultural Science, Kobe University, Nada, Kobe, 657-8501, Japan

Keywords: GR24, strigolactone, hydroxylation, sorghum, Striga, germination

*Corresponding author. Tel.: +81 788035884

E-mail address: yukihiro@kobe-u.ac.jp

${ }^{\dagger}$ Present address: Faculty of Agriculture, Tottori University, Koyama, Tottori, 680-8553, Japan 


\begin{abstract}
Bioconversion of GR24, the most widely used synthetic strigolactone (SL), by hydroponically grown sorghum (Sorghum bicolor) and biological activities of hydroxylated GR24 stereoisomers were studied. Analysis of extracts and exudates of sorghum roots previously fed with a racemic and diastereomeric mixture of GR24, using liquid chromatography-tandem mass spectrometry with multiple reaction monitoring (MRM), confirmed uptake of GR24 and suggested its conversion to mono-hydroxylated products. Two major GR24 metabolites, 7-hydroxy-GR24 and 8-hydroxy-GR24, were identified in the root extracts and exudates by direct comparison of chromatographic behavior with a series of synthetic mono-hydroxylated GR24 analogues. Separate feeding experiments with each of the GR24 stereoisomers revealed that the hydroxylated products were derived from 2'-epi-GR24, an evidence of sterical recognition of the GR24 molecule by sorghum. Trans-4-hydroxy-GR24 isomers derived from all GR24 stereoisomers were detected in the exudates as minor metabolites. The synthetic hydroxy-GR24 isomers induced germination of Striga hermonthica in decreasing order of C-8 > C-7 > C-6 > C-5 > C-4. In contrast the stereoisomers having the same configuration of orobanchol, irrespective of position of hydroxylation, induced germination of S. gesnerioides. The results confirm previous reports on structural requirements of SLs and ascribe a critical role to hydroxylation, but not to the position of the hydroxyl group in the AB part of the molecule, in induction of $S$. gesnerioides seed germination.
\end{abstract}




\section{Introduction}

Root parasitic weeds of the genera Striga, Orobanche and Phelipanche adversely affect many important food crops. ${ }^{1}$ The germination of seeds of root parasitic weeds depends on chemical signals released from host and non-host plants into the rhizosphere. Since the identification of strigol (1, Fig. 1), ${ }^{2}$ more than 10 analogous compounds have been isolated and identified from many plants as germination signals for the parasitic weeds. ${ }^{3}$ These compounds, generally known as strigolactones (SLs), share a common structure composed of a tricyclic lactone (the $\mathrm{ABC}$ part) connected via an enol ether bridge to a methylfuranone group (the D-ring). SLs were also identified as a branching factor for arbuscular mycorrhizal fungi and a new class of plant hormones that play a key role in the regulation of plant architecture. ${ }^{4}$ SLs are apocarotenoids derived from $\beta$-carotene ${ }^{5}$ via carlactone (2). ${ }^{6}$ The latter has a SLlike carbon skeleton and is synthesized by sequential reactions of three biosynthetic enzymes D27, carotenoid cleavage dioxygenase 7 (CCD7) and CCD8. ${ }^{7}$ Recently, a rice cytochrome P450 MAX1 homolog was reported to catalyze conversion of carlactone (2) to 4-deoxyorobanchol (3), which was further converted to orobanchol (4) by another MAX1 homolog. ${ }^{8}$

Fig. 1

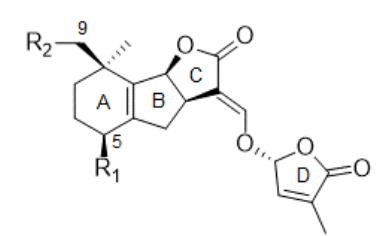

Strigol (1): $\mathrm{R}_{1}=\mathrm{OH}, \mathrm{R}_{2}=\mathrm{H}$ Sorgomol (7): $\mathrm{R}_{1}=\mathrm{H}, \mathrm{R}_{2}=\mathrm{OH}$ 5-Deoxystrigol (8): $\mathrm{R}_{1}=\mathrm{R}_{2}=\mathrm{H}$

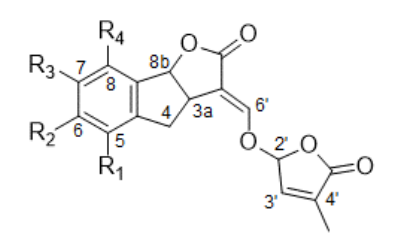

$\mathrm{GR} 24$ (5): $\mathrm{R}_{1}=\mathrm{R}_{2}=\mathrm{R}_{3}=\mathrm{R}_{4}=\mathrm{H}$ 5-HO-GR24 (11): $\mathrm{R}_{1}=\mathrm{OH}, \mathrm{R}_{2}=\mathrm{R}_{3}=\mathrm{R}_{4}=\mathrm{H}$ 6-HO-GR24 (12): $\mathrm{R}_{1}=\mathrm{H}, \mathrm{R}_{2}=\mathrm{OH}, \mathrm{R}_{3}=\mathrm{R}_{4}=\mathrm{H}$ 7-HO-GR24 (13): $\mathrm{R}_{1}=\mathrm{R}_{2}=\mathrm{H}, \mathrm{R}_{3}=\mathrm{OH}, \mathrm{R}_{4}=\mathrm{H}$ 8-HO-GR24 (14): $R_{1}=R_{2}=R_{3}=H, R_{4}=O H$

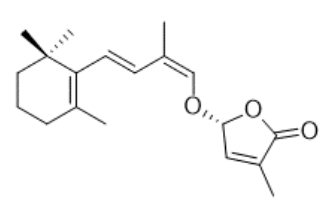

Carlactone (2)

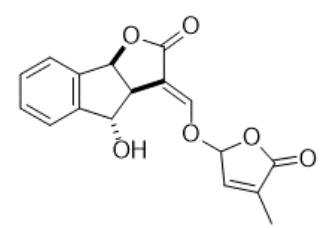

trans-4-HO-GR24 (9)

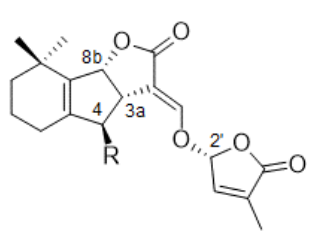

4-Deoxyorobanchol (3): $\mathrm{R}=\mathrm{H}$ Orobanchol (4): $\mathrm{R}=\mathrm{OH}$

Alectrol (6): $R=O A c$

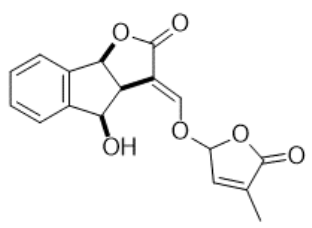

cis-4-HO-GR24 (10) 
Since naturally occurring SLs are difficult to obtain in sizeable quantities necessary for physiological studies, several SL analogues with simpler structures but retaining most of the activities were synthesized. ${ }^{9-11}$ The GR compounds, among which GR24 (5) has been widely used as the standard in germination bioassays, fulfill these criteria. However, seeds of different species showed differential response. S. gesnerioides seeds are exceptionally unresponsive to GR24 (5). The seeds germinate in response to orobanchol (4) and its acetate derivative, alectrol (6), released by roots of cowpea and red clover, a host and a non-host plant, respectively ${ }^{12,13}$. In the course of structure elucidation of orobanchol (4) and alectrol (6), it was found that the stereoisomer of GR24 having the same configuration at C-3a, C8b and C-2' as orobanchol (4) and a hydroxyl group at C-4 in the trans configuration towards the C-ring induced germination of $S$. gesnerioides seeds. ${ }^{14}$ Furthermore, it was demonstrated that the stereoisomers of 1, 4 and sorgomol (7), fulfilling the above-mentioned stereochemical requirements at C-3a and C-8b, were active in inducing germination of $S$. gesnerioides seeds thus confirming the importance of stereochemistry of the C-ring and a hydroxyl group in the AB-part of the SL molecule. ${ }^{15}$ Hydroxylation of the B-ring was also reported to enhance the activity of inducing hyphal branching in Gigaspora margarita, however, stereochemistry of the C-ring is of lesser importance. ${ }^{16}$ The importance of a hydroxyl group in the SL skeleton as a plant hormone has been unclear and solanacol (4-hydroxy-7,8dimethyl-GR24) was reported to be less active than GR24 (5) on bud-outgrowth inhibition in pea plant. ${ }^{17}$

In a preliminary trial for the bioconversion of 5-deoxystrigol (8) to sorgomol (7) by a high sorgomol-producing variety of sorghum, ${ }^{18}$ GR24 (5), as a model SL, was fed to sorghum in aquaculture. The synthetic SL was absorbed by the plant and several mono-hydroxylated products, of unknown structures, were exuded. The present work was undertaken to identify the hydroxylated products of GR24 (5) based on comparison of their chromatographic behavior with that of authentic samples in LC-MS/MS analyses. To this end, the diastereomers of GR24 derivatives hydroxylated at C-4, $-5,-6,-7$ or -8 , namely cis/trans-4-hydroxy-GR24 (trans-4-HO-GR24, 9; cis-4-HO-GR24, 10), 5-hydroxy-GR24 (5-HO-GR24, 11), 6-hydroxy-GR24 (6-HO-GR24, 12), 7-hydroxy-GR24 (7-HO-GR24, 13) and 8-hydroxy-GR24 (8- 
HO-GR24, 14) were prepared. Furthermore, the respective stereoisomers were separated by chiral HPLC and their germination-inducing activity was determined, using S. hermonthica and S. gesnerioides seeds, to further the understanding of the structure-activity relationship.

\section{Results and discussion}

\subsection{Conversion of GR24 stereoisomers by sorghum}

A racemic and diastereomeric mixture of GR24 (5) was administered to sorghum aquaculture. SLs, extracted $24 \mathrm{~h}$ later from the roots and culture filtrate were analyzed by LC-MS/MS. Total ion current (TIC) and multiple reaction monitoring (MRM) chromatograms of the root extracts and the culture filtrate are shown in Figure 2A and 2B, respectively. The TIC chromatogram indicated uptake of exogenously applied GR24 (5) by sorghum roots. However, a considerable amount of the compound was also detected in the culture filtrates. Several peaks, profiled by the MRM channel at $m / z 315>97$, were perceived in the root extracts and filtrates from cultures supplemented with GR24 (5). These peaks suggest conversion of GR24 to mono-oxygenated products with a hydroxyl group in the ABC-part of the molecule. Two metabolites were eluted at retention times $\left(t_{R} \mathrm{~s}\right) 13.2$ and $16.6 \mathrm{~min}$ in the root extracts.

Fig. 2

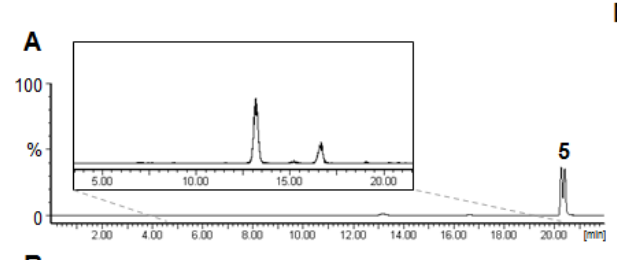

D
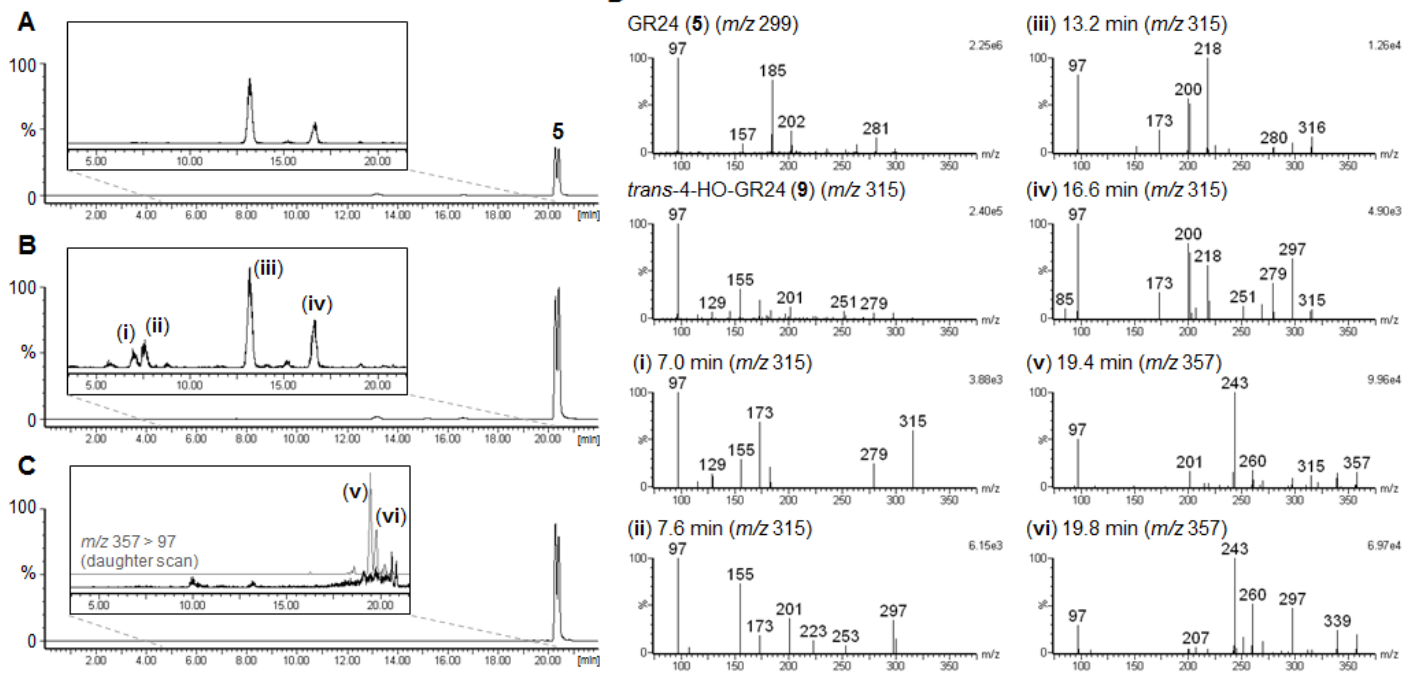
Additional two were detected at $t_{\mathrm{R}} \mathrm{s} 7.0$ and $7.6 \mathrm{~min}$ in the root exudates. The daughter ion scans at the proton adduct ion of these products together with those of authentic GR24 (5) and trans-4-HO-GR24 (9) are shown in Figure 2D. The major peaks at $t_{\mathrm{R}} 13.2 \mathrm{~min}$ (iii) and $16.6 \mathrm{~min}$ (iv) showed fragment ions at $m / z 97,173,200,201$ and 218, whereas minor ones at $t_{\mathrm{R}} 7.0 \mathrm{~min}(\mathbf{i})$ and $t_{\mathrm{R}} 7.6 \mathrm{~min}$ (ii) generated ions at $m / z 97,155,173$. The chromatographic behavior and mass fragmentation of the minor peaks coincided with those of trans-4-HO-GR24 (9). To speculate the position of hydroxylation in the products iii and iv, the crude GR24 metabolites extracted from the root exudates were treated with acetic anhydride under acidic conditions. As a result, acetylated products detected by the transition at $\mathrm{m} / z 357>97$ in the daughter ion scan with increased retention times were readily yielded (Fig. 2C, in box). In the daughter ion scan analysis at $m / z 357$, both of the acetylated products with $t_{\mathrm{R}} \mathrm{s} 19.4 \mathrm{~min}(\mathbf{v})$ and $19.8 \mathrm{~min}(\mathbf{v i})$ generated fragment ions at $\mathrm{m} / \mathrm{z} 243$ and 260 in addition to $\mathrm{m} / \mathrm{z}$ 97, the former two were greater than the corresponding fragment ions of the unacetylated metabolites (iii and iv; $m / z 201$ and 218, respectively) by 42 mass units (Fig. 2D). Since the tertiary hydroxyl group in the methyl ester of abscisic acid was hardly acetylated under the same conditions (data not shown), hydroxylation by sorghum at C-3a and $-8 \mathrm{~b}$ in GR24 was unlikely. Therefore, the metabolites with $t_{\mathrm{R}} \mathrm{s} 13.2$ and $16.6 \mathrm{~min}$ in Figure 2, exhibiting different chromatographic behavior and fragmentations from those of $\mathbf{9}$ should have the hydroxyl group at C-4 in the cis configuration towards the C-ring or in the A-ring. As observed in Figure $2 \mathrm{~A}$ and $2 \mathrm{~B}$, the conversion was modest and accordingly the available amount of GR24 metabolites was too small to be identified by spectroscopic analyses such as NMR, possible hydroxylated products with a hydroxyl group at $\mathrm{C}-4$ in the cis configuration (compound 10), C-5, -6, -7 or -8 in the A-ring of GR24 (compounds 11-14) were synthesized for direct comparison of the chromatographic behavior.

\subsection{Synthesis of hydroxylated GR24 analogues}

Diastereomers of cis-4-HO-GR24 (10) were prepared as reported previously. ${ }^{19}$ A racemic and diastereomeric mixtures of hydroxylated GR24 at C-5 (compound 11), C-6 (compound 12), C-7 
(compound 13) or C-8 (compound 14) were synthesized from the corresponding hydroxyl indan-1-ones (15) as shown in Figure 3A. After protecting the hydroxyl group in the A-ring in each hydroxyl indanone with methoxymethyl (MOM) ether, the products (16) were subjected to the established synthetic procedures of GR $24^{20}$ with some modifications. The carboxymethyl side chain was introduced into the MOM-protected hydroxyl indanones (16) by using iodoethylacetate and lithium diisopropylamide (LDA) as a base. The corresponding alkylated compounds (17), obtained in 53-72\% yields, were hydrolyzed with $1 \mathrm{M} \mathrm{NaOH}$ solution at reflux temperature. Subsequent reduction of ketone group with sodium borohydride (NBH) gave the MOM-protected tricyclic ABC-rings (18) in a modest yield (13-27\%). The compounds 18 were formylated, and then coupled with the brominated D-ring (19) to give the MOMprotected hydroxyl-GR24 isomers (20). Each racemic and diastereomeric mixture of 6-, 7- or 8-MOMOGR24 was separated by silica gel column chromatography (CC) with hexane/EtOAc to give fast- and slow- moving diastereomers. Deprotection of the MOM group with trifluoroacetic acid (TFA) in $\mathrm{CH}_{2} \mathrm{Cl}_{2}$ gave the corresponding hydroxyl GR24 diastereomers of 12-14. A racemic and diastereomeric mixture of

Fig. 3

A

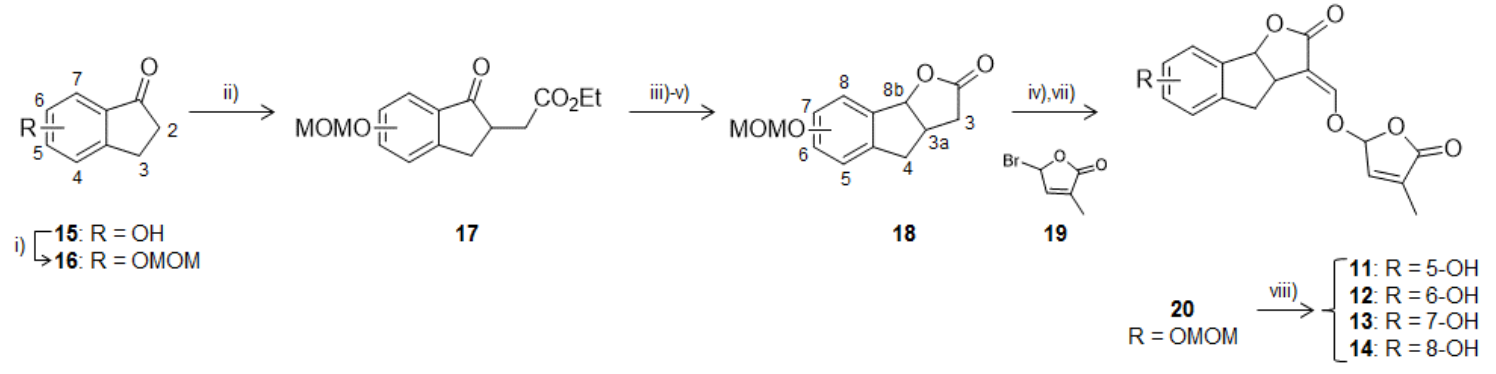

B

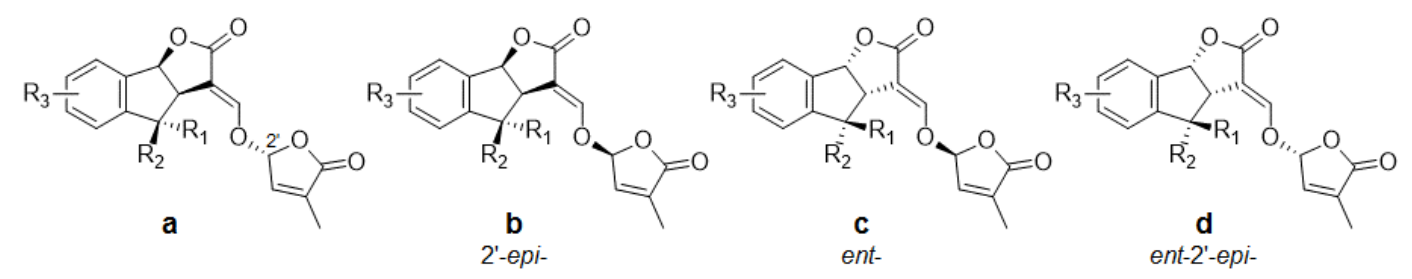

5, $\mathrm{R}_{1}=\mathrm{R}_{2}=\mathrm{R}_{3}=\mathrm{H} ; \mathbf{9}, \mathrm{R}_{1}=\mathrm{OH}, \mathrm{R}_{2}=\mathrm{R}_{3}=\mathrm{H} ; 10, \mathrm{R}_{1}=\mathrm{H}, \mathrm{R}_{2}=\mathrm{OH}, \mathrm{R}_{3}=\mathrm{H} ; 11-14, \mathrm{R}_{1}=\mathrm{R}_{2}=\mathrm{H}, \mathrm{R}_{3}=\mathrm{OH}$ 
5-HO-GR24 (11) was separated by silica gel $\mathrm{CC}$ with $\mathrm{CH}_{2} \mathrm{Cl}_{2}$ /acetone to give fast- and slow- moving diastereomers after treatment of 5-MOMO-GR24 with TFA. Each diastereomer (racemic mixture) was optically resolved with chiral column HPLC and absolute configuration of each stereoisomer (Fig. 3B) was determined by comparing its circular dichroism (CD) spectrum with those of GR24 stereoisomers (Fig. 4). ${ }^{14}$ It should be noted that protection of the hydroxyl group in hydroxy-indanones with tertbutyldimethylsilyl ether failed to introduce the alkyl moiety into the B-ring. Hydrolysis of the ester group in the compounds $\mathbf{1 7}$ under acidic conditions was unsuccessful due to concomitant deprotection of the MOM group.

Fig. 4
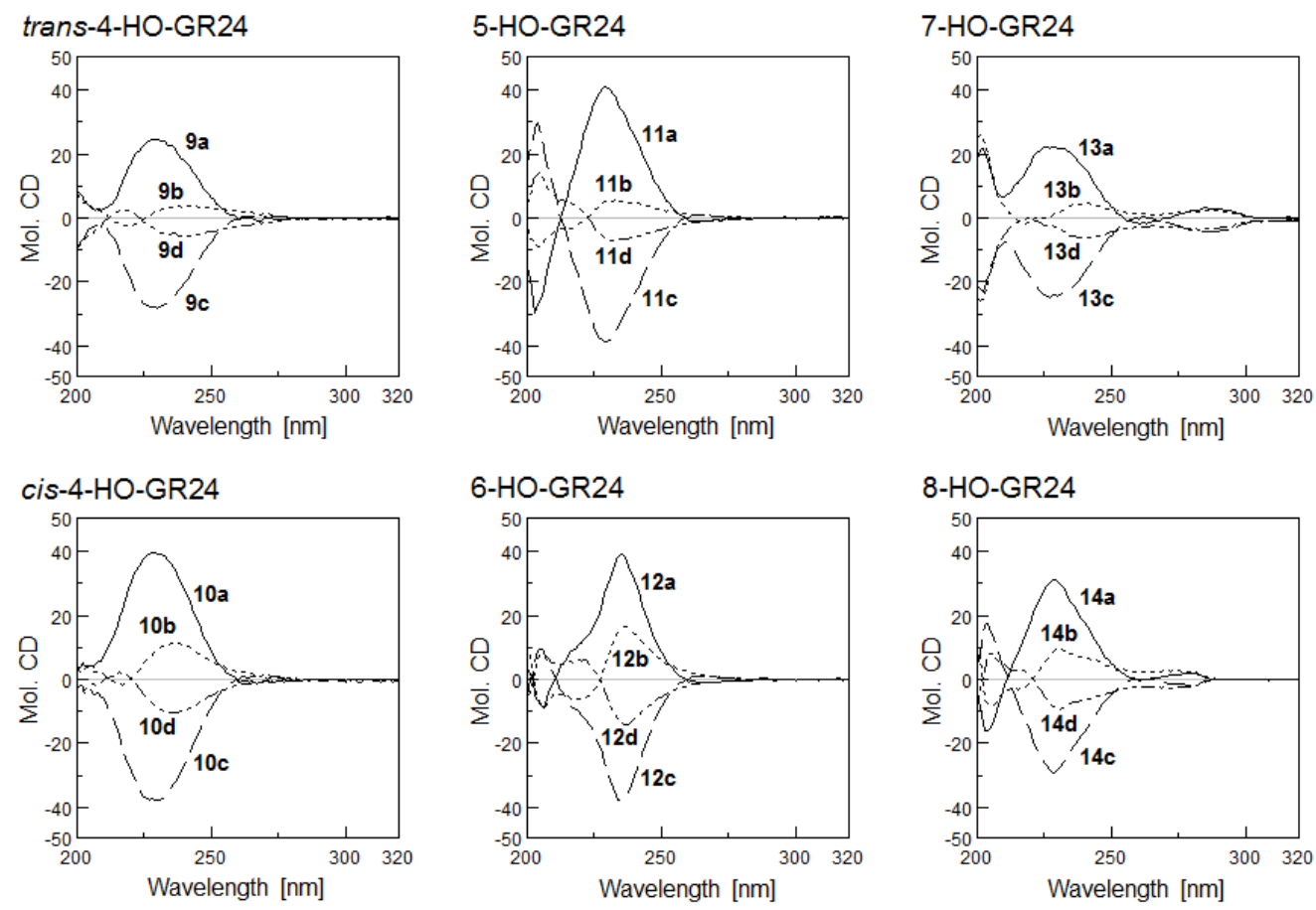

\subsection{Identification of the hydroxylated products of GR24}

Direct comparison with authentic hydroxyl GR24 isomers 9-14 indicated that the major products were hydroxylated at C-7 or C-8 while products hydroxylated at C-4 with cis orientation, C-5 or C-6 were negligible (Fig. 5A, 5B). The results indicated that hydroxylation of GR24 (5) by sorghum proceeds in a 
regioselective manner. To delineate the stereospecificity of the hydroxylation, each of the GR24 stereoisomers 5a-5d (Fig. 3B, $R_{1}=R_{2}=R_{3}=H$ ) was fed separately to sorghum aquaculture. The SLs, extracted $24 \mathrm{~h}$ later from the culture filtrate, were analyzed by the MRM channel at $m / z, 315>97$ (Fig. 5C). Based on the chromatographic behavior as well as mass spectra, the most preferred substrate was found to be 2'-epi-GR24 (5b) which was hydroxylated predominantly at C-7 but hydroxylation at C-8 was also substantial. The hydroxylated metabolite at C-7 was also detected when optically pure GR24 (5a) was administered. Trans-4-HO-GR24 (9) was a common metabolite of all the GR24 stereoisomers. Peak areas of the hydroxyl GR24 isomers 9, $\mathbf{1 3}$ and $\mathbf{1 4}$ are shown in Figure 5D. As described above, the 7- or 8-hydroxylated metabolites (13 and $\mathbf{1 4})$ were predominantly derived from 2'-epi-GR24 (5b). In contrast, the 4-hydroxylated metabolite (9) was yielded regardless of the substrate stereochemistry.

Fig. 5

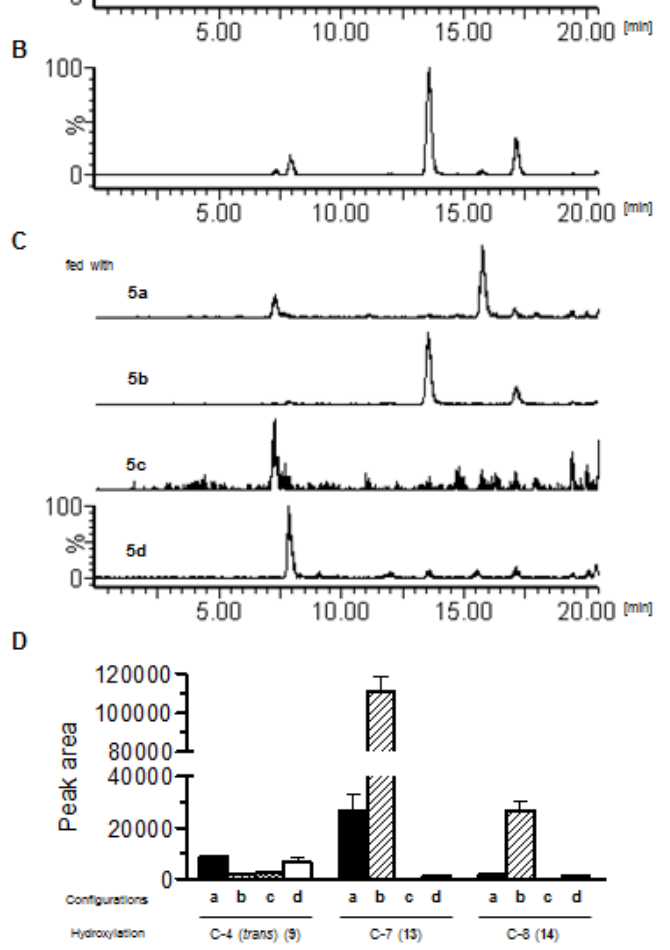

A

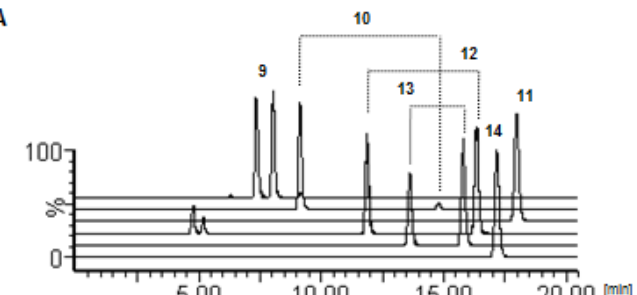




\subsection{Germination-inducing activities of hydroxylated GR24 stereoisomers}

Germination-inducing activities of stereoisomers of GR24 (5a-d) and their hydroxylated products at C-4 (9a-d and 10a-d), C-5 (11a-d), C-6 (12a-d), C-7 (13a-d), or C-8 (14a-d) on S. hermonthica and $S$. gesnerioides seeds are shown in Figure 6. Compounds with the same configuration at C-3a, C-8b and C2' as natural sorgomol (7) (configuration a, Fig. 3B) exhibited higher germination-inducing activity toward S. hermonthica than those with other stereostructures regardless of the position of the hydroxyl group. The stereochemical preference was in good agreement with the previous report for stereoisomers of natural SLs. ${ }^{15}$ Hydroxylation of GR24 did not increase germination-inducing activity in any stereoisomer except ent-8-HO-GR24 (14c). Among the hydroxylated compounds, activity decreased in the order of the hydroxylated positions at C-8 > C-7 > C-6 $>$ C-5 $>$ C-4. Taking account of the previous proposal that essential structural features of SLs required for germination-inducing activity reside in the CD-part of the molecule, ${ }^{21}$ the hydroxyl group introduced into the GR24 molecule may disturb interactions of the ligand with the receptor. This idea would be supported by less germination induction achieved by molecules with the hydroxyl group closer to the proposed bioactiphore.

Fig. 6

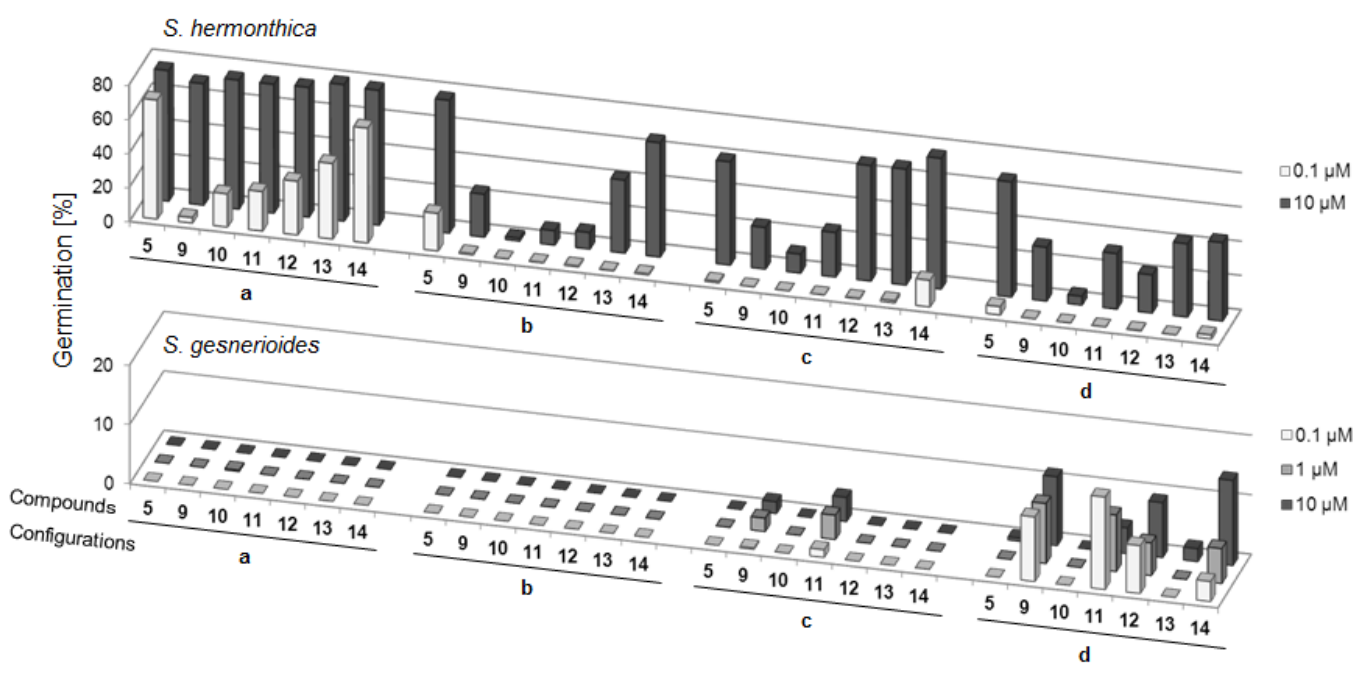


Toward S. gesnerioides seeds, isomers having the same configuration at C-3a, C-8b and C-2' as orobanchol (4) and alectrol (6) (configuration d, Fig. 3B) exhibited moderate activity, confirming the importance of the stereochemistry and a hydroxyl group for activity. ${ }^{14}$ Contrasting activity between ent2'-epi-trans-4-HO-GR24 (9d) and ent-2'-epi-cis-4-HO-GR24 (10d) demonstrates the importance of orientation of the hydroxyl group at C-4. Besides these compounds, ent-trans-4-HO-GR24 (9c) and ent-5HO-GR24 (11c) exhibited weak but appreciable activity. These results confirm previous finding that the configuration at the $\mathrm{BC}$ junction and a hydroxyl group are essential structural features required for germination induction of $S$. gesnerioides seeds. ${ }^{15}$

It has been well documented that, in plants, catabolic oxidizing enzyme activities are present and/or induced by foreign compounds. For example, the cytochrome P-450 enzymes are involved in the detoxification of xenobiotics such as herbicides, and are implicated in herbicide resistance in a number of

plant species. ${ }^{22,23}$ As reported previously, ${ }^{18}$ sorghum converted 5-deoxystrigol (8) to sorgomol (7) probably by a P-450 enzyme because uniconazole-P inhibited the conversion whereas prohexadione, a 2oxoglutarate-dependent dioxygenase inhibitor, had no inhibitory effect on the conversion. Involvement of a P-450 enzyme in hydroxylation of GR24 is uncertain due to ambiguous effect of uniconazole-P on the conversion. Functional analysis of the enzyme catalyzing hydroxylation of 5-deoxystrigol (8) at C-9, whose cloning and expression are ongoing, will clarify whether hydroxylation of GR24 (5) is catalyzed by the same enzyme as that for sorgomol formation.

\section{Conclusion}

The present work demonstrated that exogenously applied GR24 (5) was absorbed by sorghum roots, hydroxylated in a regioselective and stereospecific manner, and exuded out of the roots. The most preferred substrate was 2'-epi-GR24 (5b), which was converted into 2'-epi-7-HO-GR24 (13b) and 2'-epi8-HO-GR24 (14b). Minor products included trans-4-HO-GR24 stereoisomers (9a-d) and 7-HO-GR24 (13a). However, the identity of the enzyme involved in the hydroxylation needs to be ascertained. The 
structure-activity data on germination of S. hermonthica seeds confirmed bioactiphore of SLs composed of the D-ring, the enol ether linkage and the $\alpha, \beta$-unsaturated carbonyl. Further, the results confirm the importance of hydroxylation, but not the position of the hydroxyl group in the AB part of the molecule, in induction of $S$. gesnerioides seed germination.

\section{Experimental section}

\subsection{General remarks}

A racemic and distereomeric mixture of GR24 (5) was prepared as described previously ${ }^{20}$ and separated into four stereoisomers by silica gel column chromatography (CC) followed by HPLC equipped with a chiral column as described previously. ${ }^{14}$ Other chemicals were purchased from Wako Pure Chemical Industries, Ltd. (Osaka, Japan), Sigma-Aldrich Japan (Tokyo, Japan) or Tokyo Chemical Industry, Ltd. (TCI, Tokyo, Japan). Melting points were determined on a Yanaco melting point apparatus (MP-S3 model, Tokyo, Japan). ${ }^{1} \mathrm{H}$ NMR (300 MHz) and ${ }^{13} \mathrm{C}$ NMR (75 MHz) spectra were recorded in $\mathrm{CDCl}_{3}$ except hydroxyl GR24s which were in acetone- $d_{6}$, on a JNM-AL 300 spectrometer (JEOL, Tokyo, Japan) using tetramethylsilane (TMS) as an internal standard, and chemical shifts are shown in $\delta$ (ppm). MS spectra were obtained on a JEOL JMS-700 mass spectrometer in the EI mode. CD spectra were recorded in acetonitrile with a J-805 spectropolarimeter (JASCO Corp., Tokyo, Japan).

\subsection{Synthesis of trans4-hydroxy-GR24 (9) and cis-4-hydroxy-GR24 (10)}

A 2'-epimeric mixture of trans-4-hydroxy-GR24 (9) was prepared and stereoisomers were separated as previously reported. ${ }^{14}$ Cis-4-hydroxy-GR24 (cis-4-HO-GR24, 10) was synthesized as reported previously. ${ }^{19}$ Diastereomers of [2-(trimethylsilyl)ethoxy]methyl (SEM)-protected 10 were separated by silica gel CC eluting with hexane/EtOAc (1:1) to EtOAc to give fast-moving and slowmoving diastereomers. Fast-moving diastereomer $(9.4 \mathrm{mg})$ was deprotected with trifluoroacetic acid (TFA) $(0.2 \mathrm{~mL})$ in $\mathrm{CH}_{2} \mathrm{Cl}_{2}(1 \mathrm{~mL})$ to give $\mathbf{1 0 a} / \mathbf{c}(3.7 \mathrm{mg}, 56 \%)$, which was separated by chiral HPLC on a 
CHIRALPAK IC $(250 \mathrm{~mm} \times 10 \mathrm{~mm}, 5 \mu \mathrm{m})$ by using hexane/EtOH $(1: 1)$ as the mobile phase at a flow rate of $1.0 \mathrm{~mL} / \mathrm{min}$. The column effluent was monitored at $233 \mathrm{~nm}$ to obtain ent-cis-4-HO-GR24 (10c) and cis-4-HO-GR24 (10a) at $71 \mathrm{~min}$ and $78 \mathrm{~min}$, respectively. The slow-moving diastereomer of SEMprotected 10 was similarly deprotected and then separated under the same conditions to obtain ent-2'-epicis-4-HO-GR24 (10d) and 2'-epi-cis-4-HO-GR24 (10b) at $24 \mathrm{~min}$ and $34 \mathrm{~min}$, respectively. CD, $\lambda_{\text {ext }}$, $(\Delta \varepsilon): \mathbf{1 0 a}(c 0.000041), 267(-1.2), 229(+39.8), 206(+3.6), 202(+5.1) \mathrm{nm} ; \mathbf{1 0 b}(c 0.000085), 236(+11.2)$, 217 (-1.7), 204 (+3.9), 201 (+2.5) nm; 10c (c 0.000021), 266 (+1.1), $227(-37.9), 205(-3.5), 203(-5.3)$ $\mathrm{nm} ; \mathbf{1 0 d}(c$ 0.000064), $238(-10.6), 216(+2.2), 203(-3.4), 200(-2.3) \mathrm{nm}$.

\subsection{Synthesis of 5-, 6-, 7-, or 8-hydroxy-GR24 (11-14)}

\subsubsection{Synthesis of MOM-protected hydroxyl 1-indanones (16)}

\subsubsection{4-(Methoxymethoxy)-2,3-dihydro-1H-inden-1-one (4-MOMO-16)}

4-Hydroxy-1-indanone (4-HO-15) $(0.5 \mathrm{~g}, 3.4 \mathrm{mmol})$ was suspended in $\mathrm{CH}_{2} \mathrm{Cl}_{2}(0.5 \mathrm{~mL})$ at $0{ }^{\circ} \mathrm{C}$, $N, N$-diisopropylethylamine (DIPEA, $3.0 \mathrm{~mL}, 17.6 \mathrm{mmol}$ ) and chloromethyl methyl ether (MOMCl, 1.6 $\mathrm{mL}, 21.3 \mathrm{mmol}$ ) were added to the reaction mixture, and stirred at room temperature (RT) for $2 \mathrm{~h}$. The resulting mixture was diluted with water and extracted with $\mathrm{CH}_{2} \mathrm{Cl}_{2}$. The organic layer was washed with brine and dried over $\mathrm{MgSO}_{4}$ and concentrated. The residue was purified by silica gel CC eluting with hexane/EtOAc (1:1) to EtOAc to give 4-MOMO-16 (0.56 g, 86\% yield) as an orange solid. Mp. $46-48{ }^{\circ} \mathrm{C}$; ${ }^{1} \mathrm{H}$ NMR: $\delta$ 2.67-2.71 (m, 2H, 3- $\left.\mathrm{CH}_{2}\right), 3.05-3.09\left(\mathrm{~m} .2 \mathrm{H}, 2-\mathrm{CH}_{2}\right), 3.51\left(\mathrm{~s}, 3 \mathrm{H}, \mathrm{OCH}_{3}\right), 5.27(\mathrm{~s}, 2 \mathrm{H}$, $\left.\mathrm{OCH}_{2} \mathrm{O}\right), 7.28(\mathrm{dd}, J=7.8$ and $1.5 \mathrm{~Hz}, 1 \mathrm{H}, 5-\mathrm{H}), 7.33(\mathrm{dd}, J=7.8$ and $6.9 \mathrm{~Hz}, 1 \mathrm{H}, 6-\mathrm{H}), 7.41(\mathrm{dd}, J=6.9$ and $1.5 \mathrm{~Hz}, 1 \mathrm{H}, 7-\mathrm{H}) ;{ }^{13} \mathrm{C}$ NMR: $\delta 22.6,36.1,56.2,94.4,116.6,118.7,128.8,138.8,144.4,154.7,207.0$; HREIMS m/z $192.0788[\mathrm{M}]^{+}\left(\mathrm{C}_{11} \mathrm{H}_{12} \mathrm{O}_{3}\right.$ requires 192.0786).

\subsubsection{5-(Methoxymethoxy)-2,3-dihydro-1H-inden-1-one (5-MOMO-16)}


5-MOMO-16 (0.59 g) was prepared from 5-HO-15 (0.5 g) as described in Section 4.3.1.1 with a yield of $91 \%$ as a white solid. Mp. 57-59 ${ }^{\circ} \mathrm{C}$, ${ }^{1} \mathrm{H}$ NMR: $\delta$ 2.65-2.69 (m, 2H, 3-CH $), 3.07-3.11(\mathrm{~m} .2 \mathrm{H}, 2-$ $\left.\mathrm{CH}_{2}\right), 3.49\left(\mathrm{~s}, 3 \mathrm{H}, \mathrm{OCH}_{3}\right), 5.25\left(\mathrm{~s}, 2 \mathrm{H}, \mathrm{OCH}_{2} \mathrm{O}\right), 6.99(\mathrm{dd}, J=8.4$ and $1.5 \mathrm{~Hz}, 1 \mathrm{H}, 6-\mathrm{H}), 7.08(\mathrm{~d}, J=1.5$ $\mathrm{Hz}, 1 \mathrm{H}, 4-\mathrm{H}), 7.69$ (d, $J=8.4 \mathrm{~Hz}, 1 \mathrm{H}, 7-\mathrm{H}) ;{ }^{13} \mathrm{C}$ NMR: $\delta 25.8,36.4,56.3,94.1,112.2,116.7,125.3$, 131.2, 157.9, 162.7, 205.4; HREIMS m/z 192.0796 [M] $]^{+}\left(\mathrm{C}_{11} \mathrm{H}_{12} \mathrm{O}_{3}\right.$ requires 192.0786).

\subsubsection{6-(Methoxymethoxy)-2,3-dihydro-1H-inden-1-one (6-MOMO-16)}

6-MOMO-16 (2.4 g) was prepared from 6-HO-15 (2.0 g) as described in Section 4.3.1.1 with a yield of $92 \%$ as a yellow solid. Mp. $61-63{ }^{\circ} \mathrm{C} ;{ }^{1} \mathrm{H}$ NMR: $\delta$ 2.69-2.73 (m, 2H, 3-CH $), 3.06-3.10$ (m. 2H, 2$\left.\mathrm{CH}_{2}\right), 3.47\left(\mathrm{~s}, 3 \mathrm{H}, \mathrm{OCH}_{3}\right), 5.19\left(\mathrm{~s}, 2 \mathrm{H}, \mathrm{OCH}_{2} \mathrm{O}\right), 7.28(\mathrm{dd}, J=8.4$ and $2.4 \mathrm{~Hz}, 1 \mathrm{H}, 5-\mathrm{H}), 7.37-7.40(\mathrm{~m}, 2 \mathrm{H}$, 4- and 7-H); ${ }^{13}$ C NMR: $\delta 25.1,36.9,56.0,94.5,109.1,124.6,127.4,138.2,148.7,156.7,206.8$; HREIMS $m / z 192.0783[\mathrm{M}]^{+}\left(\mathrm{C}_{11} \mathrm{H}_{12} \mathrm{O}_{3}\right.$ requires 192.0786).

\subsubsection{7-(Methoxymethoxy)-2,3-dihydro-1H-inden-1-one (7-MOMO-16)}

7-MOMO-16 (2.07 g) was prepared from 7-HO-15 (2.10 g) as described in Section 4.3.1.1 with a yield of $74 \%$ as a pale yellow solid. Mp. $69-71{ }^{\circ} \mathrm{C} ;{ }^{1} \mathrm{H}$ NMR: $\delta$ 2.65-2.72 (m, 2H, 3-CH 2 ), 3.06-3.09 (m. $\left.2 \mathrm{H}, 2-\mathrm{CH}_{2}\right), 3.52\left(\mathrm{~s}, 3 \mathrm{H}, \mathrm{OCH}_{3}\right), 5.34\left(\mathrm{~s}, 2 \mathrm{H}, \mathrm{OCH}_{2} \mathrm{O}\right), 7.04$ and $7.07(\mathrm{~d}, J=8.2 \mathrm{~Hz}$, each 1H, 4- and 6H), $7.49(\mathrm{dd}, J=8.2$ and $8.2 \mathrm{~Hz}, 1 \mathrm{H}, 5-\mathrm{H}) ;{ }^{13} \mathrm{C} \mathrm{NMR}: \delta 25.4,36.8,56.4,94.5,112.9,119.6,125.9,136.1$, 155.4, 157.6, 204.5; HREIMS m/z 192.0788 $[\mathrm{M}]^{+}\left(\mathrm{C}_{11} \mathrm{H}_{12} \mathrm{O}_{3}\right.$ requires 192.0786).

\subsubsection{Synthesis of ethyl ester of MOM-protected 2-(hydroxy-1-oxo-2,3-dihydro- $1 H$-inden-2-yl) acetate (17)}

\subsubsection{Ethyl 2-[4-(methoxymethoxy)-1-oxo-2,3-dihydro-1H-inden-2-yl]acetate (4-MOMO-17)}

Diisopropylamine $(320 \mu \mathrm{L}, 2.3 \mathrm{mmol})$ was dissolved in tetrahydrofuran (THF) at $0{ }^{\circ} \mathrm{C}$, and $n$ $\mathrm{BuLi}(880 \mu \mathrm{L}, 2.3 \mathrm{mmol})$ was added and stirred for $20 \mathrm{~min}$. The reaction mixture was then cooled at -78 
${ }^{\circ} \mathrm{C}$ and a solution of 4-MOMO-16 $(0.4 \mathrm{~g}, 2.1 \mathrm{mmol})$ in THF $(2 \mathrm{~mL})$ was added dropwise to the mixture with stirring at $-78{ }^{\circ} \mathrm{C}$ for $10 \mathrm{~min}$. Iodoethylacetate $(270 \mu \mathrm{L}, 2.3 \mathrm{mmol})$ was added to the reaction mixture and stirred for $30 \mathrm{~min}$. The mixture was warmed to $\mathrm{RT}$, saturated $\mathrm{NH}_{4} \mathrm{Cl}$ aq. was added and subsequently extracted with ether. The organic layer was washed with brine, dried over $\mathrm{MgSO}_{4}$ and concentrated. The residue was purified by silica gel CC eluting with hexane/EtOAc (3:1) to EtOAc to give 4-MOMO-17 (0.42 g, 72\% yield) as a brown oil. ${ }^{1} \mathrm{H} \mathrm{NMR}: \delta 1.22\left(\mathrm{t}, J=7.2 \mathrm{~Hz}, 3 \mathrm{H}, \mathrm{CO}_{2} \mathrm{CH}_{2} \mathrm{CH}_{3}\right)$, $2.65\left(\mathrm{dd}, J=17.4\right.$ and $\left.7.8 \mathrm{~Hz}, 1 \mathrm{H}, \mathrm{CH}_{2} \mathrm{CO}_{2} \mathrm{Et}\right), 2.77(\mathrm{dd}, J=17.4$ and $4.2 \mathrm{~Hz}, 1 \mathrm{H}, 3-\mathrm{H}), 2.96(\mathrm{dd}, J=17.4$ and $\left.4.2 \mathrm{~Hz}, 1 \mathrm{H}, \mathrm{CH}_{2} \mathrm{CO}_{2} \mathrm{Et}\right), 2.98-3.04(\mathrm{~m}, 1 \mathrm{H}, 2-\mathrm{H}), 3.42(\mathrm{dd}, J=17.4$ and $8.1 \mathrm{~Hz}, 3-\mathrm{H}), 3.50(\mathrm{~s}, 3 \mathrm{H}$, $\left.\mathrm{OCH}_{3}\right), 4.14\left(\mathrm{q}, J=7.2 \mathrm{~Hz}, 2 \mathrm{H}, \mathrm{CO}_{2} \mathrm{CH}_{2} \mathrm{CH}_{3}\right), 5.26\left(\mathrm{~s}, 2 \mathrm{H}, \mathrm{OCH}_{2} \mathrm{O}\right), 7.28(\mathrm{dd}, J=7.8$ and $1.5 \mathrm{~Hz}, 1 \mathrm{H}, 5-$ H), $7.33(\mathrm{dd}, J=7.8$ and $7.2 \mathrm{~Hz}, 1 \mathrm{H}, 6-\mathrm{H}), 7.41(\mathrm{dd}, J=7.2$ and $1.5 \mathrm{~Hz}, 1 \mathrm{H}, 7-\mathrm{H}) ;{ }^{13} \mathrm{C}$ NMR: $\delta 14.1,29.7$, 35.3, 43.4, 56.2, 60.7, 94.3, 116.8, 119.0, 129.0, 138.0, 142.5, 154.5, 171.9, 206.7; HREIMS m/z 278.1151 [M] $]^{+}\left(\mathrm{C}_{15} \mathrm{H}_{18} \mathrm{O}_{5}\right.$ requires 278.1154).

\subsubsection{Ethyl 2-[5-(methoxymethoxy)-1-oxo-2,3-dihydro-1H-inden-2-yl]acetate (5-MOMO-17)}

5-MOMO-17 (0.33 g) was prepared from 5-MOMO-16 (0.4 g) as described in Section 4.3.2.1 with a yield of $57 \%$ as a brown oil. ${ }^{1} \mathrm{H}$ NMR: $\delta 1.23\left(\mathrm{t}, J=7.2 \mathrm{~Hz}, 3 \mathrm{H}, \mathrm{CO}_{2} \mathrm{CH}_{2} \mathrm{CH}_{3}\right), 2.58(\mathrm{dd}, J=16.5$ and $\left.8.7 \mathrm{~Hz}, 1 \mathrm{H}, \mathrm{CH}_{2} \mathrm{CO}_{2} \mathrm{Et}\right), 2.84(\mathrm{dd}, J=17.1$ and $4.5 \mathrm{~Hz}, 1 \mathrm{H}, 3-\mathrm{H}), 2.96(\mathrm{dd}, J=16.5$ and $4.2 \mathrm{~Hz}, 1 \mathrm{H}$, $\left.\mathrm{CH}_{2} \mathrm{CO}_{2} \mathrm{Et}\right), 2.98-3.04(\mathrm{~m}, 1 \mathrm{H}, 2-\mathrm{H}), 3.40(\mathrm{dd}, J=17.1$ and $7.5 \mathrm{~Hz}, 3-\mathrm{H}), 3.49\left(\mathrm{~s}, 3 \mathrm{H}, \mathrm{OCH}_{3}\right), 4.14(\mathrm{q}, J=$ $\left.7.2 \mathrm{~Hz}, 2 \mathrm{H}, \mathrm{CO}_{2} \mathrm{CH}_{2} \mathrm{CH}_{3}\right), 5.25\left(\mathrm{~s}, 2 \mathrm{H}, \mathrm{OCH}_{2} \mathrm{O}\right), 7.01(\mathrm{dd}, J=8.1$ and $1.8 \mathrm{~Hz}, 1 \mathrm{H}, \mathrm{H}-6), 7.06(\mathrm{~d}, J=1.8$ $\mathrm{Hz}, 1 \mathrm{H}, \mathrm{H}-4), 7.70$ (d, $J=8.1 \mathrm{~Hz}, 1 \mathrm{H}, 7-\mathrm{H}) ;{ }^{13} \mathrm{C} \mathrm{NMR}: \delta 14.1,33.0,35.4,43.7,56.3,60.7,94.1,112.1$, 116.8, 125.6, 130.4, 156.0, 162.9, 172.0, 205.0; HREIMS m/z 278.1145 [M] $]^{+}\left(\mathrm{C}_{15} \mathrm{H}_{18} \mathrm{O}_{5}\right.$ requires 278.1154).

\subsubsection{Ethyl 2-[6-(methoxymethoxy)-1-oxo-2,3-dihydro-1H-inden-2-yl]acetate (6-MOMO-17)}


6-MOMO-17 (0.5 g) was prepared from 6-MOMO-16 (0.5 g) as described in Section 4.3.2.1 with a yield of $68 \%$ as a yellow oil. ${ }^{1} \mathrm{H}$ NMR: $\delta 1.22\left(\mathrm{t}, J=7.2 \mathrm{~Hz}, 3 \mathrm{H}, \mathrm{CO}_{2} \mathrm{CH}_{2} \mathrm{CH}_{3}\right), 2.62(\mathrm{dd}, J=16.5$ and $\left.8.4 \mathrm{~Hz}, 1 \mathrm{H}, \mathrm{CH}_{2} \mathrm{CO}_{2} \mathrm{Et}\right), 2.82(\mathrm{dd}, J=16.8$ and $4.2 \mathrm{~Hz}, 1 \mathrm{H}, 3-\mathrm{H}), 2.96(\mathrm{dd}, J=16.5$ and $3.9 \mathrm{~Hz}, 1 \mathrm{H}$, $\left.\mathrm{CH}_{2} \mathrm{CO}_{2} \mathrm{Et}\right), 3.03(\mathrm{~m}, 1 \mathrm{H}, 2-\mathrm{H}), 3.38(\mathrm{dd}, J=16.8$ and $7.8 \mathrm{~Hz}, 3-\mathrm{H}), 3.47\left(\mathrm{~s}, 3 \mathrm{H}, \mathrm{OCH}_{3}\right), 4.14(\mathrm{q}, J=7.2$ $\left.\mathrm{Hz}, 2 \mathrm{H}, \mathrm{CO}_{2} \mathrm{CH}_{2} \mathrm{CH}_{3}\right), 5.20\left(\mathrm{~s}, 2 \mathrm{H}, \mathrm{OCH}_{2} \mathrm{O}\right), 7.27(\mathrm{dd}, J=8.4$ and $2.1 \mathrm{~Hz}, 1 \mathrm{H}, 5-\mathrm{H}), 7.37(\mathrm{~d}, J=8.4 \mathrm{~Hz}$, $1 \mathrm{H}, 4-\mathrm{H}), 7.40(\mathrm{~d}, J=2.1 \mathrm{~Hz}, 1 \mathrm{H}, 7-\mathrm{H}) ;{ }^{13} \mathrm{C} \mathrm{NMR}: \delta 14.1,32.3,35.3,44.2,56.1,60.7,94.5,109.4,124.8$, 127.2, 137.5, 146.8, 156.9, 171.9, 206.5; HREIMS m/z 278.1151 [M] $]^{+}\left(\mathrm{C}_{15} \mathrm{H}_{18} \mathrm{O}_{5}\right.$ requires 278.1154).

\subsubsection{Ethyl 2-[7-(methoxymethoxy)-1-oxo-2,3-dihydro-1H-inden-2-yl]acetate (7-MOMO-17)} 7-MOMO-17 (0.32 g) was prepared from 7-MOMO-16 (0.42 g) as described in Section 4.3.2.1 with a yield of $53 \%$ as a pale yellow oil. ${ }^{1} \mathrm{H}$ NMR: $\delta 1.23\left(\mathrm{t}, J=7.2 \mathrm{~Hz}, 3 \mathrm{H}, \mathrm{CO}_{2} \mathrm{CH}_{2} \mathrm{CH}_{3}\right), 2.55(\mathrm{dd}, J=$ 17.1 and $\left.9.3 \mathrm{~Hz}, 1 \mathrm{H}, \mathrm{CH}_{2} \mathrm{CO}_{2} \mathrm{Et}\right), 2.84(\mathrm{dd}, J=17.1$ and $4.5 \mathrm{~Hz}, 1 \mathrm{H}, 3-\mathrm{H}), 2.97(\mathrm{dd}, J=17.1$ and $3.9 \mathrm{~Hz}$, $\left.1 \mathrm{H}, \mathrm{CH}_{2} \mathrm{CO}_{2} \mathrm{Et}\right), 2.96-3.06(\mathrm{~m}, 1 \mathrm{H}, 2-\mathrm{H}), 3.41(\mathrm{dd}, J=17.1$ and $8.1 \mathrm{~Hz}, 3-\mathrm{H}), 3.52\left(\mathrm{~s}, 3 \mathrm{H}, \mathrm{OCH}_{3}\right), 4.14$ (q, $\left.J=7.2 \mathrm{~Hz}, 2 \mathrm{H}, \mathrm{CO}_{2} \mathrm{CH}_{2} \mathrm{CH}_{3}\right), 5.33\left(\mathrm{~s}, 2 \mathrm{H}, \mathrm{OCH}_{2} \mathrm{O}\right), 7.04$ and $7.05(\mathrm{~d}, J=7.8 \mathrm{~Hz}$, each 1H, 4- and 6H), $7.50(\mathrm{dd}, J=7.8$ and $7.8 \mathrm{~Hz}, 1 \mathrm{H}, 5-\mathrm{H}) ;{ }^{13} \mathrm{C}$ NMR: $\delta 14.1,32.6,35.4,43.9,56.4,60.6,94.5,113.0$, 119.4, 125.1, 136.3, 155.5, 155.5, 172.1, 204.0; HREIMS $m / z .278 .1162[\mathrm{M}]^{+}\left(\mathrm{C}_{15} \mathrm{H}_{18} \mathrm{O}_{5}\right.$ requires 278.1154).

\subsubsection{Synthesis of MOM-protected hydroxyl tricyclic lactone (18)}

\subsubsection{5-(Methoxymethoxy)-3,3a,4,8b-tetrahydro-2H-indeno[1,2b]furan-2-one (5-MOMO-18)}

The ester 4-MOMO-17 (0.26 g, $0.92 \mathrm{mmol})$ was dissolved in $1 \mathrm{M} \mathrm{NaOH}$ aq. (3 mL) and stirred at reflux temp. for $3 \mathrm{~h}$. The reaction mixture was cooled to $0{ }^{\circ} \mathrm{C}$. Sodium borohydride $(0.21 \mathrm{~g}, 5.5 \mathrm{mmol})$ was then added to the mixture and stirred at RT for $24 \mathrm{~h}$ and cooled to $0{ }^{\circ} \mathrm{C}$. The mixture was acidified with $1 \mathrm{M} \mathrm{HCl}$ aq. to $\mathrm{pH} \mathrm{2,} \mathrm{and} \mathrm{the} \mathrm{reaction} \mathrm{mixture} \mathrm{was} \mathrm{stirred} \mathrm{at} \mathrm{RT}$ for $16 \mathrm{~h}$. The resulting mixture was diluted with water and extracted with $\mathrm{CHCl}_{3}$. The organic layer was washed with brine, dried over 
$\mathrm{MgSO}_{4}$ and concentrated. The residue was purified by silica gel CC eluting with hexane/EtOAc $(1: 1)$ to give 5-MOMO-18 (27 mg, 13\% yield) as a yellow oil. ${ }^{1} \mathrm{H}$ NMR: $\delta 2.41(\mathrm{dd}, J=18.3$ and $5.5 \mathrm{~Hz}, 1 \mathrm{H}, 3-$ H), $2.85(\mathrm{dd}, J=16.7$ and $3.1 \mathrm{~Hz}, 1 \mathrm{H}, \mathrm{H}-4), 2.92(\mathrm{dd}, J=18.3$ and $9.7 \mathrm{~Hz}, \mathrm{H}-3), 3.26(\mathrm{dd}, J=16.7$ and $8.3 \mathrm{~Hz}, 1 \mathrm{H}, 4-\mathrm{H}), 3.30-3.42(\mathrm{~m}, 1 \mathrm{H}, 3 \mathrm{a}-\mathrm{H}), 3.48\left(\mathrm{~s}, 3 \mathrm{H}, \mathrm{OCH}_{3}\right), 5.21\left(\mathrm{~s}, 2 \mathrm{H}, \mathrm{OCH}_{2} \mathrm{O}\right), 5.88(\mathrm{~d}, J=6.9 \mathrm{~Hz}$, 1H, 8b-H), 6.97-7.29 (m, 3H, 6-, 7- and 8-H). ${ }^{13} \mathrm{C}$ NMR: $\delta 35.0,35.8,37.1,56.1,87.9,94.3,114.5,119.4$, 129.3, 131.3, 140.6, 153.9, 176.9; HREIMS m/z 234.0900 [M] $]^{+}\left(\mathrm{C}_{13} \mathrm{H}_{14} \mathrm{O}_{4}\right.$ requires 234.0892).

\subsubsection{6-(Methoxymethoxy)-3,3a,4,8b-tetrahydro- $2 H$-indeno[1,2b]furan-2-one (6-MOMO-18)}

6-MOMO-18 (59 mg) was prepared from 5-MOMO-17 (372 $\mathrm{mg})$ as described in Section 4.3.3.1 with a yield of $19 \%$ as a white solid. Mp. $86-88{ }^{\circ} \mathrm{C} ;{ }^{1} \mathrm{H}$ NMR: $\delta 2.40(\mathrm{dd}, J=18.0$ and $5.3 \mathrm{~Hz}, 1 \mathrm{H}, 3-\mathrm{H})$, $2.85(\mathrm{dd}, J=16.3$ and $3.3 \mathrm{~Hz}, 1 \mathrm{H}, \mathrm{H}-4), 2.90(\mathrm{dd}, J=18.0$ and $9.6 \mathrm{~Hz}, \mathrm{H}-3), 3.28(\mathrm{dd}, J=16.3$ and 8.4 $\mathrm{Hz}, 1 \mathrm{H}, 4-\mathrm{H}), 3.30-3.40(\mathrm{~m}, 1 \mathrm{H}, 3 \mathrm{a}-\mathrm{H}), 3.48\left(\mathrm{~s}, 3 \mathrm{H}, \mathrm{OCH}_{3}\right), 5.17\left(\mathrm{~s}, 2 \mathrm{H}, \mathrm{OCH}_{2} \mathrm{O}\right), 5.83(\mathrm{~d}, J=6.9 \mathrm{~Hz}$, 1H, 8b-H), $6.94(\mathrm{~s}, 1 \mathrm{H}, 5-\mathrm{H}), 6.95(\mathrm{~d}, J=7.8 \mathrm{~Hz}, 7-\mathrm{H}), 7.38(\mathrm{~d}, J=7.8 \mathrm{~Hz}, 1 \mathrm{H}, 8-\mathrm{H}) ;{ }^{13} \mathrm{C}$ NMR: $\delta 35.8$, 37.8, 38.1, 56.0, 87.3, 94.4, 112.2, 116.2, 127.2, 132.2, 144.5, 158.9, 176.9; HREIMS m/z 234.0898 [M] $^{+}$ $\left(\mathrm{C}_{13} \mathrm{H}_{14} \mathrm{O}_{4}\right.$ requires 234.0892).

\subsubsection{7-(Methoxymethoxy)-3,3a,4,8b-tetrahydro-2H-indeno[1,2b]furan-2-one (7-MOMO-18)}

7-MOMO-18 (0.21 g) was prepared from 6-MOMO-17 (1.0 g) as described in Section 4.3.3.1 with a yield of $20 \%$ as a yellow solid. Mp. $54-55{ }^{\circ} \mathrm{C} ;{ }^{1} \mathrm{H}$ NMR: $\delta 2.38(\mathrm{dd}, J=18.0$ and $6.0 \mathrm{~Hz}, 1 \mathrm{H}, 3-\mathrm{H})$, $2.82(\mathrm{dd}, J=15.9$ and $3.3 \mathrm{~Hz}, 1 \mathrm{H}, 4-\mathrm{H}), 2.89(\mathrm{dd}, J=18.0$ and $9.9 \mathrm{~Hz}, 3-\mathrm{H}), 3.25(\mathrm{dd}, J=15.9$ and 8.4 $\mathrm{Hz}, 1 \mathrm{H}, 4-\mathrm{H}), 3.33-3.41(\mathrm{~m}, 1 \mathrm{H}, 3 \mathrm{a}-\mathrm{H}), 3.48\left(\mathrm{~s}, 3 \mathrm{H}, \mathrm{OCH}_{3}\right), 5.16$ and 5.17 (AB pattern, $J=18.0 \mathrm{~Hz}, 2 \mathrm{H}$, $\left.\mathrm{OCH}_{2} \mathrm{O}\right), 5.85(\mathrm{~d}, J=7.2 \mathrm{~Hz}, 1 \mathrm{H}, 8 \mathrm{~b}-\mathrm{H}), 7.02(\mathrm{dd}, J=8.4$ and $2.4 \mathrm{~Hz}, 1 \mathrm{H}, 6-\mathrm{H}), 7.16(\mathrm{~d}, J=2.4 \mathrm{~Hz}, 1 \mathrm{H}$, 8-H), 7.17 (d, $J=8.4 \mathrm{~Hz}, 1 \mathrm{H}, 5-\mathrm{H}) ;{ }^{13} \mathrm{C}$ NMR: $\delta 35.6,37.1,37.8,56.0,87.6,94.6,113.5,118.9,126.0$, 135.4, 140.0, 157.0, 176.9; HREIMS m/z, 234.0887 [M] $]^{+}\left(\mathrm{C}_{13} \mathrm{H}_{14} \mathrm{O}_{4}\right.$ requires 234.0892). 


\subsubsection{8-(Methoxymethoxy)-3,3a,4,8b-tetrahydro-2H-indeno[1,2b]furan-2-one (8-MOMO-18)}

8-MOMO-18 (0.41 g) was prepared from 7-MOMO-17 (1.8 g) as described in Section 4.3.3.1 with a yield of $27 \%$ yield as a white solid. Mp. $97-99{ }^{\circ} \mathrm{C} ;{ }^{1} \mathrm{H}$ NMR: $\delta 2.47(\mathrm{dd}, J=18.0$ and $3.6 \mathrm{~Hz}, 1 \mathrm{H}$, 3-H), 2.83-2.98 (m, 2H, 3- and 4-H), 3.26-3.40 (m, 2H, 3a- and 4-H), 3.50 (s, 3H, $\left.\mathrm{OCH}_{3}\right), 5.26$ (s, $2 \mathrm{H}$, $\left.\mathrm{OCH}_{2} \mathrm{O}\right), 6.01(\mathrm{~d}, J=6.6 \mathrm{~Hz}, 1 \mathrm{H}, 8 \mathrm{~b}-\mathrm{H}), 6.89(\mathrm{~d}, J=7.5 \mathrm{~Hz}, 1 \mathrm{H}, 5-$ or $7-\mathrm{H}), 6.98(\mathrm{~d}, J=8.1 \mathrm{~Hz}, 1 \mathrm{H}, 5$ - or 7-H), $7.30(\mathrm{dd}, J=8.1$ and $7.5 \mathrm{~Hz}, 1 \mathrm{H}, 6-\mathrm{H}),{ }^{13} \mathrm{C}$ NMR: $\delta 35.8,37.2,38.7,56.2,85.7,94.1,112.4,118.2$, 127.5, 131.9, 145.4, 155.0, 176.9; HREIMS $m / z 234.0889[\mathrm{M}]^{+}\left(\mathrm{C}_{13} \mathrm{H}_{14} \mathrm{O}_{4}\right.$ requires 234.0892).

\subsubsection{Synthesis of MOM-protected hydroxyl GR24s (20)}

\subsubsection{1. (E)-5-(Methoxymethoxy)-3-\{[(4-methyl-5-oxo-2,5-dihydrofuran-2-yl)oxy]methylene)-} 3,3a,4,8b-tetrahydro-2H-indeno[1,2-b]furan-2-one (5-MOMO-20)

A solution of 5-MOMO-18 $(34 \mathrm{mg}, 0.15 \mathrm{mmol})$ in THF $(1 \mathrm{~mL})$ was cooled to $0{ }^{\circ} \mathrm{C}$, and $\mathrm{NaH}$ (55\% in mineral oil, $35 \mathrm{mg}, 1.46 \mathrm{mmol})$ and methyl formate $(270 \mu \mathrm{L}, 4.40 \mathrm{mmol})$ were added to the solution. The reaction mixture, stirred at RT for $5.5 \mathrm{~h}$, was diluted with brine and extracted with EtOAc. The organic layer was dried over $\mathrm{MgSO}_{4}$ and concentrated. The crude mixture of enolate was used without further purification. A solution of 5-bromo-3-methylfuran-2(5H)-one (19, $20 \mathrm{mg}, 0.11 \mathrm{mmol})$ in dimethoxyethane (DME) $(1 \mathrm{~mL})$ was added to the crude enolate. $\mathrm{K}_{2} \mathrm{CO}_{3}(32 \mathrm{mg}, 0.23 \mathrm{mmol})$ was added to the mixture and stirred at RT for $16 \mathrm{~h}$. The resulting mixture was diluted with brine and extracted with EtOAc. The organic layer was dried over $\mathrm{MgSO}_{4}$ and concentrated. The residue was purified by silica gel CC eluting with hexane/EtOAc (1:1) to EtOAc to give 5-MOMO-20 (18.9 mg, 46\% yield) as a yellow oil of racemic and diastereomeric mixture. ${ }^{1} \mathrm{H}$ NMR: $\delta 2.04\left(\mathrm{~s}, 3 \mathrm{H}, 4^{\prime}-\mathrm{CH}_{3}\right), 3.05(\mathrm{~d}, J=17.1 \mathrm{~Hz}, 1 \mathrm{H}, 4-\mathrm{H})$, $3.37(\mathrm{dd}, J=17.1$ and $8.4 \mathrm{~Hz}, 1 \mathrm{H}, 4-\mathrm{H}), 3.47\left(\mathrm{~s}, 3 \mathrm{H}, \mathrm{OCH}_{3}\right), 3.90-4.00(\mathrm{~m}, 1 \mathrm{H}, 3 \mathrm{a}-\mathrm{H}), 5.20(\mathrm{~s}, 2 \mathrm{H}$, $\left.\mathrm{OCH}_{2} \mathrm{O}\right), 5.94(\mathrm{~d}, J=7.8 \mathrm{~Hz}, 1 \mathrm{H}, 8 \mathrm{~b}-\mathrm{H}), 6.19\left(\mathrm{~m}, 1 \mathrm{H}, 2^{\prime}-\mathrm{H}\right), 6.97$ (m, 1H, 3'-H), 7.04 (d, J = 8.1 Hz, 1H, 6-H), 7.14 (d, $J=7.2 \mathrm{~Hz}, 1 \mathrm{H}, 8-\mathrm{H}), 7.24$ (dd, $J=8.1$ and $7.2 \mathrm{~Hz}, 1 \mathrm{H}, 7-\mathrm{H}), 7.49$ (d, $J=2.4 \mathrm{~Hz}, 1 \mathrm{H}, 6$ '-H);

${ }^{13} \mathrm{C}$ NMR: $\delta 10.7,34.6 / 34.7,38.7,56.1,86.1,94.2,100.5 / 100.6,113.3,114.4,119.3 / 119.4,129.2 / 129.3$, 
131.5, 136.0, 140.6, 140.9, 150.9/151.0, 153.6, 170.2, 171.2/171.3; HREIMS m/z 358.1054 [M] ${ }^{+}$

$\left(\mathrm{C}_{19} \mathrm{H}_{18} \mathrm{O}_{7}\right.$ requires 358.1053).

\subsubsection{2. (E)-6-(Methoxymethoxy)-3-\{[(4-methyl-5-oxo-2,5-dihydrofuran-2-yl)oxy]methylene)-}

\section{3,3a,4,8b-tetrahydro-2H-indeno[1,2-b]furan-2-one (6-MOMO-20)}

A racemic and diastereomeric mixture of 6-MOMO-20 was prepared from 6-MOMO-18 (31 mg) as described in Section 4.3.4.1. The mixture was separated by silica gel CC eluting with hexane/EtOAc (1:1) to obtain fast-moving diastereomer $(14.3 \mathrm{mg}, 31 \%$ yield) and slow-moving diastereomer (10.8 mg, $23 \%$ yield) as pale yellow oils. The fast-moving diastereomer 6-MOMO-20a/c. ${ }^{1} \mathrm{H}$ NMR: $\delta 2.04(\mathrm{dd}, J=$ 1.5 and $\left.1.5 \mathrm{~Hz}, 3 \mathrm{H}, 4^{\prime}-\mathrm{CH}_{3}\right), 3.06(\mathrm{~d}, J=16.8$ and $3.3 \mathrm{~Hz}, 1 \mathrm{H}, 4-\mathrm{H}), 3.40$ (dd, $J=16.8$ and $9.3 \mathrm{~Hz}, 1 \mathrm{H}, 4-$ $\mathrm{H}), 3.47\left(\mathrm{~s}, 3 \mathrm{H}, \mathrm{OCH}_{3}\right), 3.91-3.99(\mathrm{~m}, 1 \mathrm{H}, 3 \mathrm{a}-\mathrm{H}), 5.17\left(\mathrm{~s}, 2 \mathrm{H}, \mathrm{OCH}_{2} \mathrm{O}\right), 5.90(\mathrm{~d}, J=7.8 \mathrm{~Hz}, 1 \mathrm{H}, 8 \mathrm{~b}-\mathrm{H})$, $6.18\left(\mathrm{dq}, J=1.5\right.$ and $\left.1.5 \mathrm{~Hz}, 1 \mathrm{H}, 2^{\prime}-\mathrm{H}\right), 6.90(\mathrm{br} \mathrm{s}, 1 \mathrm{H}, 5-\mathrm{H}), 6.95(\mathrm{dd}, J=8.4$ and $1.8 \mathrm{~Hz}, 1 \mathrm{H}, 7-\mathrm{H}), 6.97$ (dq, $J=1.5$ and $\left.1.5 \mathrm{~Hz}, 1 \mathrm{H}, 3^{\prime}-\mathrm{H}\right), 7.40(\mathrm{~d}, J=8.4 \mathrm{~Hz}, 1 \mathrm{H}, 8-\mathrm{H}), 7.47\left(\mathrm{~d}, J=2.4 \mathrm{~Hz}, 1 \mathrm{H}, 6^{\prime}-\mathrm{H}\right){ }^{13} \mathrm{C}$ NMR: $\delta 10.7,37.4,39.3,56.0,85.5,94.3,100.5,111.8,113.4,116.3,127.3,132.3,136.0,140.9,144.5$, 150.8, 158.9, 170.2, 171.3; HREIMS $m / z$ 358.1052 $[\mathrm{M}]^{+}\left(\mathrm{C}_{19} \mathrm{H}_{18} \mathrm{O}_{7}\right.$ requires 358.1053). The slow-moving diastereomer 6-MOMO-20b/d. ${ }^{1} \mathrm{H}$ NMR: $\delta 2.05\left(\mathrm{dd}, J=1.5\right.$ and $\left.1.5 \mathrm{~Hz}, 3 \mathrm{H}, 4^{\prime}-\mathrm{CH}_{3}\right), 3.05(\mathrm{~d}, J=16.8$ and $3.3 \mathrm{~Hz}, 1 \mathrm{H}, 4-\mathrm{H}), 3.38(\mathrm{dd}, J=16.8$ and $9.3 \mathrm{~Hz}, 1 \mathrm{H}, 4-\mathrm{H}), 3.47\left(\mathrm{~s}, 3 \mathrm{H}, \mathrm{OCH}_{3}\right), 3.91-3.96(\mathrm{~m}, 1 \mathrm{H}, 3 \mathrm{a}-$ H), $5.17\left(\mathrm{~s}, 2 \mathrm{H}, \mathrm{OCH}_{2} \mathrm{O}\right), 5.90(\mathrm{~d}, J=7.8 \mathrm{~Hz}, 1 \mathrm{H}, 8 \mathrm{~b}-\mathrm{H}), 6.18\left(\mathrm{dq}, J=1.5\right.$ and $\left.1.5 \mathrm{~Hz}, 1 \mathrm{H}, 2^{\prime}-\mathrm{H}\right), 6.90$ (br s, $1 \mathrm{H}, 5-\mathrm{H}), 6.94(\mathrm{dd}, J=8.4$ and $2.4 \mathrm{~Hz}, 1 \mathrm{H}, 7-\mathrm{H}), 6.97\left(\mathrm{dq}, J=1.5\right.$ and $\left.1.5 \mathrm{~Hz}, 1 \mathrm{H}, 3^{\prime}-\mathrm{H}\right), 7.39(\mathrm{~d}, J=$ 8.4 Hz, 1H, 8-H), 7.47 (d, $\left.J=2.4 \mathrm{~Hz}, 1 \mathrm{H}, 6{ }^{\prime}-\mathrm{H}\right) ;{ }^{13} \mathrm{C}$ NMR: $\delta 10.7,37.5,39.3,56.0,85.5,94.3,100.6$, 111.9, 113.5, 116.3, 127.2, 132.2, 136.0, 140.9, 144.5, 150.8, 158.9, 170.2, 171.3; HREIMS m/z 358.1055 $[\mathrm{M}]^{+}\left(\mathrm{C}_{19} \mathrm{H}_{18} \mathrm{O}_{7}\right.$ requires 358.1053).

\subsubsection{3. (E)-7-(Methoxymethoxy)-3-\{[(4-methyl-5-oxo-2,5-dihydrofuran-2-yl)oxy]methylene)-} 3,3a,4,8b-tetrahydro-2H-indeno[1,2-b]furan-2-one (7-MOMO-20) 
A racemic and diastereomeric mixture of 7-MOMO-20 was prepared from 7-MOMO-18 (150 $\mathrm{mg}$ ) as described in Section 4.3.4.1. The mixture was separated by silica gel CC eluting with hexane/EtOAc (1:1) to obtain fast-moving diastereomer (60.3 mg, 26\% yield) and slow-moving diastereomer (61.8 mg, 27\% yield) as pale yellow solids. The fast-moving diastereomer 7-MOMO-20a/c. Mp. $148-149{ }^{\circ} \mathrm{C} ;{ }^{1} \mathrm{H}$ NMR: $\delta 2.04\left(\mathrm{dd}, J=1.5\right.$ and $\left.1.5 \mathrm{~Hz}, 3 \mathrm{H}, 4^{\prime}-\mathrm{CH}_{3}\right), 3.04(\mathrm{~d}, J=16.5$ and $3.3 \mathrm{~Hz}, 1 \mathrm{H}$, 4-H), $3.36(\mathrm{dd}, J=16.5$ and $9.0 \mathrm{~Hz}, 1 \mathrm{H}, 4-\mathrm{H}), 3.47\left(\mathrm{~s}, 3 \mathrm{H}, \mathrm{OCH}_{3}\right), 3.96$ (dddd, $J=9.0,7.8,3.3$ and 2.4 $\mathrm{Hz}, 1 \mathrm{H}, 3 \mathrm{a}-\mathrm{H}), 5.15$ and $5.17\left(\mathrm{AB}\right.$ pattern, $\left.J=18.9 \mathrm{~Hz}, 2 \mathrm{H}, \mathrm{OCH}_{2} \mathrm{O}\right), 5.91(\mathrm{~d}, J=7.8 \mathrm{~Hz}, 1 \mathrm{H}, 8 \mathrm{~b}-\mathrm{H}), 6.18$ (dq, $J=1.5$ and $\left.1.5 \mathrm{~Hz}, 1 \mathrm{H}, 2^{\prime}-\mathrm{H}\right), 6.97$ (dq, $J=1.5$ and $\left.1.5 \mathrm{~Hz}, 1 \mathrm{H}, 3^{\prime}-\mathrm{H}\right), 7.02$ (dd, $J=8.4$ and $2.4 \mathrm{~Hz}$, 1H, 6-H), $7.13(\mathrm{~d}, J=8.4 \mathrm{~Hz}, 1 \mathrm{H}, 5-\mathrm{H}), 7.18(\mathrm{~d}, J=2.4 \mathrm{~Hz}, 1 \mathrm{H}, 8-\mathrm{H}), 7.48\left(\mathrm{~d}, J=2.4 \mathrm{~Hz}, 1 \mathrm{H}, 6{ }^{\prime}-\mathrm{H}\right) ;{ }^{13} \mathrm{C}$ NMR: $\delta$ 10.7, 36.5, 39.4, 56.0, 85.8, 94.6, 100.5, 113.2, 113.4, 119.1, 125.8, 135.6, 136.0, 140.1, 140.8, 150.9, 156.9, 170.2, 171.2; HREIMS $m / z$ 358.1048 $[\mathrm{M}]^{+}\left(\mathrm{C}_{19} \mathrm{H}_{18} \mathrm{O}_{7}\right.$ requires 358.1053). The slow-moving diastereomer 7-MOMO-20b/d. Mp. 122-125 ${ }^{\circ} \mathrm{C} ;{ }^{1} \mathrm{H}$ NMR: $\delta 2.04\left(\mathrm{dd}, J=1.5\right.$ and $1.5 \mathrm{~Hz}, 3 \mathrm{H}, 4^{\prime}-\mathrm{CH}_{3}$ ), $3.03(\mathrm{~d}, J=16.8$ and $3.0 \mathrm{~Hz}, 1 \mathrm{H}, 4-\mathrm{H}), 3.35(\mathrm{dd}, J=16.8$ and $9.0 \mathrm{~Hz}, 1 \mathrm{H}, 4-\mathrm{H}), 3.47\left(\mathrm{~s}, 3 \mathrm{H}, \mathrm{OCH}_{3}\right), 3.95$ (dddd, $J=9.0,7.8,3.0$ and $2.4 \mathrm{~Hz}, 1 \mathrm{H}, 3 \mathrm{a}-\mathrm{H}), 5.15$ and $5.17\left(\mathrm{AB}\right.$ pattern, $\left.J=18.9 \mathrm{~Hz}, 2 \mathrm{H}, \mathrm{OCH}_{2} \mathrm{O}\right), 5.91$ (d, $J=7.8 \mathrm{~Hz}, 1 \mathrm{H}, 8 \mathrm{~b}-\mathrm{H}), 6.17\left(\mathrm{dq}, J=1.5\right.$ and $\left.1.5 \mathrm{~Hz}, 1 \mathrm{H}, 2^{\prime}-\mathrm{H}\right), 6.97$ (dq, $J=1.5$ and $1.5 \mathrm{~Hz}, 1 \mathrm{H}, 3^{\prime}-$ H), $7.01(\mathrm{dd}, J=8.4$ and $2.4 \mathrm{~Hz}, 1 \mathrm{H}, 6-\mathrm{H}), 7.13(\mathrm{~d}, J=8.4 \mathrm{~Hz}, 1 \mathrm{H}, 5-\mathrm{H}), 7.17(\mathrm{~d}, J=2.4 \mathrm{~Hz}, 1 \mathrm{H}, 8-\mathrm{H})$, $7.48\left(\mathrm{~d}, J=2.4 \mathrm{~Hz}, 1 \mathrm{H}, 6{ }^{\prime}-\mathrm{H}\right) ;{ }^{13} \mathrm{C}$ NMR: $\delta 10.8,36.6,39.4,56.0,85.8,94.6,100.6,113.3,113.4,119.1$, 125.9, 135.6, 136.0, 140.0, 140.9, 150.9, 156.9, 170.2, 171.2; HREIMS $m / z 358.1055[\mathrm{M}]^{+}\left(\mathrm{C}_{19} \mathrm{H}_{18} \mathrm{O}_{7}\right.$ requires 358.1053).

\subsubsection{4. (E)-8-(Methoxymethoxy)-3-\{[(4-methyl-5-oxo-2,5-dihydrofuran-2-yl)oxy $]$ methylene)- 3,3a,4,8b-tetrahydro-2H-indeno[1,2-b]furan-2-one (8-MOMO-20)}

A racemic and diastereomeric mixture of 8-MOMO-20 was prepared from 8-MOMO-18 (132 $\mathrm{mg}$ ) as described in Section 4.3.4.1 The mixture was separated by silica gel CC eluting with hexane/EtOAc (1:1) to obtain fast-moving diastereomer (28.1 mg, 12\% yield) as a pale yellow solid and 
slow-moving diastereomer (48.7 mg, $21 \%$ yield) as yellow gum. The fast-moving diastereomer 8MOMO-20a/c. Mp. $181-184{ }^{\circ} \mathrm{C} ;{ }^{1} \mathrm{H}$ NMR: $\delta 2.04$ (s, 3H, 4'-CH ), 3.05 (d, $J=16.8$ and $\left.3.6 \mathrm{~Hz}, 1 \mathrm{H}, 4-\mathrm{H}\right)$, $3.42(\mathrm{dd}, J=16.8$ and $9.6 \mathrm{~Hz}, 1 \mathrm{H}, 4-\mathrm{H}), 3.50\left(\mathrm{~s}, 3 \mathrm{H}, \mathrm{OCH}_{3}\right), 3.89-3.97(\mathrm{~m}, 1 \mathrm{H}, 3 \mathrm{a}-\mathrm{H}), 5.26(\mathrm{~s}, 2 \mathrm{H}$, $\left.\mathrm{OCH}_{2} \mathrm{O}\right), 6.07$ (d, J=7.5 Hz, 1H, 8b-H), 6.20 (br s, 1H, 2'-H), 6.86 (d, J= 7.5 Hz, 1H, 5- or 7-H), 6.96 (d, $J=7.8 \mathrm{~Hz}, 5-$ or 7-H), 6.98 (br s, 1H, 3'-H), 7.29 (dd, $J=7.8$ and $7.5 \mathrm{~Hz}, 1 \mathrm{H}, 6-\mathrm{H}), 7.49$ (d, $J=2.4$ $\left.\mathrm{Hz}, 1 \mathrm{H}, 6{ }^{\prime}-\mathrm{H}\right) ;{ }^{13} \mathrm{C}$ NMR: $\delta 10.7,38.1,38.6,56.1,84.1,94.0,100.6,112.1,113.5,118.1,127.5,131.9$, 135.8, 141.0, 145.2, 150.7, 155.0, 170.2, 171.3; HREIMS m/z 358.1047 [M] $]^{+}\left(\mathrm{C}_{19} \mathrm{H}_{18} \mathrm{O}_{7}\right.$ requires 358.1053). The slow-moving diastereomer 8-MOMO-20b/d. ${ }^{1} \mathrm{H}$ NMR: $\delta 2.04$ (s, 3H, 4'-CH $\left.{ }_{3}\right), 3.06$ (d, $J$ $=17.1$ and $3.9 \mathrm{~Hz}, 1 \mathrm{H}, 4-\mathrm{H}), 3.44(\mathrm{dd}, J=17.1$ and $9.3 \mathrm{~Hz}, 1 \mathrm{H}, 4-\mathrm{H}), 3.51\left(\mathrm{~s}, 3 \mathrm{H}, \mathrm{OCH}_{3}\right), 3.90-3.98(\mathrm{~m}$, 1H, 3a-H), 5.26 (s, 2H, OCH $2 \mathrm{O}), 6.07$ (d, $J=7.5 \mathrm{~Hz}, 1 \mathrm{H}, 8 \mathrm{~b}-\mathrm{H}), 6.19$ (br s, 1H, 2'-H), 6.86 (d, $J=7.5$ Hz, 1H, 5- or 7-H), 6.97 (d, J=7.5 Hz, 5- or 7-H), 6.98 (br s, 1H, 3'-H), 7.29 (dd, J = 7.5 and 7.5 Hz, 1H, 6-H), $7.49\left(\mathrm{~d}, J=2.1 \mathrm{~Hz}, 1 \mathrm{H}, 6{ }^{\prime}-\mathrm{H}\right) ;{ }^{13} \mathrm{C}$ NMR: $\delta 10.7,38.1,38.7,56.2,84.1,94.0,100.5,112.2,113.5$, 118.0, 127.6, 131.9, 136.0, 140.9, 145.1, 150.6, 155.1, 170.2, 171.3; HREIMS m/z 358.1061 [M] ${ }^{+}$ $\left(\mathrm{C}_{19} \mathrm{H}_{18} \mathrm{O}_{7}\right.$ requires 358.1053).

\subsubsection{Deprotection of MOM group}

\subsubsection{1. (E)-5-Hydroxy-3-\{[(4-methyl-5-oxo-2,5-dihydrofuran-2-yl)oxy]methylene)-3,3a,4,8b- tetrahydro- $2 H$-indeno[1,2-b]furan-2-one (5-hydroxy-GR24, 11)}

Trifluoroacetic acid (TFA, $0.5 \mathrm{~mL}$ ) was added to a solution of 5-MOMO-20 (14 mg, $0.04 \mathrm{mmol})$ in $\mathrm{CH}_{2} \mathrm{Cl}_{2}(1 \mathrm{~mL})$, and the mixture was stirred at $\mathrm{RT}$ for $1.5 \mathrm{~h}$. The solvent was evaporated and then the residue was purified by silica gel CC eluting with hexane/EtOAc (1:3) to give a racemic and diastereomeric mixture of 5-hydroxy-GR24 (11) (8.8 $\mathrm{mg}, 72 \%$ yield) as a brown oil. The mixture was further separated by silica gel $\mathrm{CC}$ with 2-3\% acetone in $\mathrm{CH}_{2} \mathrm{Cl}_{2}$ to give fast-moving diastereomer (4.2 $\mathrm{mg})$ and slow-moving diastereomer $(2.6 \mathrm{mg})$ as a yellow solid. The fast-moving diastereomer $\mathbf{1 1 b} / \mathbf{d}$. Mp. $185-188{ }^{\circ} \mathrm{C} ;{ }^{1} \mathrm{H} \mathrm{NMR}, \delta 2.05\left(\mathrm{dd}, J=1.5\right.$ and $\left.1.5 \mathrm{~Hz}, 3 \mathrm{H}, 4{ }^{\prime}-\mathrm{CH}_{3}\right), 3.03(\mathrm{dd}, J=16.8$ and $3.0 \mathrm{~Hz}, 1 \mathrm{H}, 4-$ 
H), $3.34(\mathrm{dd}, J=16.8$ and $9.3 \mathrm{~Hz}, 1 \mathrm{H}, 4-\mathrm{H}), 3.97$ (dddd, $J=9.3,7.8,3.0$ and $2.4 \mathrm{~Hz}, 1 \mathrm{H}, 3 \mathrm{a}-\mathrm{H}), 5.95(\mathrm{~d}, J$ $=7.8 \mathrm{~Hz}, 1 \mathrm{H}, 8 \mathrm{~b}-\mathrm{H}), 6.18\left(\mathrm{dq}, J=1.5\right.$ and $\left.1.5 \mathrm{~Hz}, 1 \mathrm{H}, 2^{\prime}-\mathrm{H}\right), 6.77(\mathrm{~d}, J=8.1 \mathrm{~Hz}, 1 \mathrm{H}, 6-\mathrm{H}), 6.97(\mathrm{dq}, J=$ 1.5 and $\left.1.5 \mathrm{~Hz}, 1 \mathrm{H}, 3^{\prime}-\mathrm{H}\right), 7.08$ (d, $\left.J=7.5 \mathrm{~Hz}, 1 \mathrm{H}, 8-\mathrm{H}\right), 7.18$ (dd, $J=8.1$ and $\left.7.5 \mathrm{~Hz}, 1 \mathrm{H}, 7-\mathrm{H}\right), 7.49$ (d, $J$ $\left.=2.4 \mathrm{~Hz}, 1 \mathrm{H}, 6{ }^{\prime}-\mathrm{H}\right) ;{ }^{13} \mathrm{C} \mathrm{NMR}, \delta 10.8,34.1,38.8,86.1,100.6,113.3,115.9,118.6,128.8,129.3,136.1$, 140.9, 141.0, 151.1, 152.3, 170.3, 171.3; HREIMS $m / z 314.0786[\mathrm{M}]^{+}\left(\mathrm{C}_{17} \mathrm{H}_{14} \mathrm{O}_{6}\right.$ requires 314.0790). EIMS m/z (rel. int.) $314[\mathrm{M}]^{+}$(17), $296\left[\mathrm{M}-\mathrm{H}_{2} \mathrm{O}\right]^{+}$(1), 252 (2), 217 (17), 200 (38), 172 (2), 171 (4), 131 (4), 115 (12), 97 (100). The slow-moving diastereomer 11a/c. Mp. 137-140 ${ }^{\circ} \mathrm{C} ;{ }^{1} \mathrm{H}$ NMR, $\delta 2.05(\mathrm{dd}, J=$ 1.5 and $\left.1.5 \mathrm{~Hz}, 3 \mathrm{H}, 4^{\prime}-\mathrm{CH}_{3}\right), 3.05$ (dd, $J=16.8$ and $\left.3.3 \mathrm{~Hz}, 1 \mathrm{H}, 4-\mathrm{H}\right), 3.36$ (dd, $J=16.8$ and $9.3 \mathrm{~Hz}, 1 \mathrm{H}$, 4-H), 3.98 (dddd, $J=9.3,7.8,3.3$ and $2.4 \mathrm{~Hz}, 1 \mathrm{H}, 3 \mathrm{a}-\mathrm{H}), 5.95$ (d, $J=7.8 \mathrm{~Hz}, 1 \mathrm{H}, 8 \mathrm{~b}-\mathrm{H}), 6.15$ (dq, $J=1.5$ and $\left.1.5 \mathrm{~Hz}, 1 \mathrm{H}, 2^{\prime}-\mathrm{H}\right), 6.76(\mathrm{~d}, J=7.5 \mathrm{~Hz}, 1 \mathrm{H}, 6-\mathrm{H}), 6.97\left(\mathrm{dq}, J=1.5\right.$ and $\left.1.5 \mathrm{~Hz}, 1 \mathrm{H}, 3^{\prime}-\mathrm{H}\right), 7.10(\mathrm{~d}, J=$ $7.2 \mathrm{~Hz}, 1 \mathrm{H}, 8-\mathrm{H}), 7.18(\mathrm{dd}, J=7.5$ and $7.2 \mathrm{~Hz}, 1 \mathrm{H}, 7-\mathrm{H}), 7.49$ (d, $\left.J=2.4 \mathrm{~Hz}, 1 \mathrm{H}, 6^{\prime}-\mathrm{H}\right) ;{ }^{13} \mathrm{C}$ NMR, $\delta$ $10.8,34.0,38.9,86.1,100.6,113.1,115.8,118.7,128.8,129.4,136.1,140.9,141.1,151.1,152.2,170.3$, 171.3; HREIMS m/z 314.0780 [M] $]^{+}\left(\mathrm{C}_{17} \mathrm{H}_{14} \mathrm{O}_{6}\right.$ requires 314.0790). EIMS m/z (rel. int.) $314[\mathrm{M}]^{+}$(24), $296\left[\mathrm{M}-\mathrm{H}_{2} \mathrm{O}\right]^{+}$(4), 252 (6), 217 (30), 200 (52), 172 (6), 171 (6), 131 (3), 115 (9), 97 (100).

\subsubsection{2. (E)-6-Hydroxy-3-\{[(4-methyl-5-oxo-2,5-dihydrofuran-2-yl)oxy]methylene)-3,3a,4,8b- tetrahydro- $2 H$-indeno[1,2-b]furan-2-one (6-hydroxy-GR24, 12)}

Each diastereomer of 6-MOMO-20 was deprotected under the same conditions as described in Section 4.3.5.1. 6-Hydroxy-GR24 (12a/c), $3.8 \mathrm{mg}$ from $14.3 \mathrm{mg}$ of 6-MOMO-20a/c (30\% yield), as a white solid. Mp. $214-216{ }^{\circ} \mathrm{C} ;{ }^{1} \mathrm{H}$ NMR (acetone- $d_{6}$ ), $\delta 1.98$ (dd, $J=1.5$ and $1.5 \mathrm{~Hz}, 3 \mathrm{H}, 4^{\prime}-\mathrm{CH}_{3}$ ), 2.99 (dd, $J=16.8$ and $3.0 \mathrm{~Hz}, 1 \mathrm{H}, 4-\mathrm{H}), 3.33(\mathrm{dd}, J=16.8$ and $9.3 \mathrm{~Hz}, 1 \mathrm{H}, 4-\mathrm{H}), 3.97$ (dddd, $J=9.3,7.8,3.0$ and $2.7 \mathrm{~Hz}, 1 \mathrm{H}, 3 \mathrm{a}-\mathrm{H}), 5.86(\mathrm{~d}, J=7.8 \mathrm{~Hz}, 1 \mathrm{H}, 8 \mathrm{~b}-\mathrm{H}), 6.57\left(\mathrm{dq}, J=1.5\right.$ and $\left.1.5 \mathrm{~Hz}, 1 \mathrm{H}, 2^{\prime}-\mathrm{H}\right), 6.68$ (s, $1 \mathrm{H}, 5-\mathrm{H}), 6.76(\mathrm{~d}, J=8.4 \mathrm{~Hz}, 1 \mathrm{H}, 7-\mathrm{H}), 7.28(\mathrm{~d}, J=8.4 \mathrm{~Hz}, 1 \mathrm{H}, 8-\mathrm{H}), 7.33(\mathrm{dq}, J=1.5$ and $1.5 \mathrm{~Hz}, 1 \mathrm{H}$, 3'-H), $7.56\left(\mathrm{~d}, J=2.7 \mathrm{~Hz}, 1 \mathrm{H}, 6{ }^{\prime}-\mathrm{H}\right) ;{ }^{13} \mathrm{C}$ NMR (acetone- $\left.d_{6}\right), \delta 10.5,37.9,40.4,86.1,102.2,112.0,113.9$, $115.7,127.9,131.5,135.5,143.2,145.9,152.4,160.0,171.3,171.4 ;$ HREIMS $m / z 314.0787[\mathrm{M}]^{+}$ 
$\left(\mathrm{C}_{17} \mathrm{H}_{14} \mathrm{O}_{6}\right.$ requires 314.0790); EIMS m/z (rel. int.) $314[\mathrm{M}]^{+}$(18), 217 (22), 200 (17), 199 (10), 189 (19), 171 (45), 145 (54), 115 (31), 97 (100). 6-Hydroxy-GR24 (12b/d), 4.3 mg from 10.7 mg of 6-MOMO20b/d (45\% yield), as a white solid. Mp. $223-225{ }^{\circ} \mathrm{C} ;{ }^{1} \mathrm{H}$ NMR (acetone- $\left.d_{6}\right), \delta 1.98(\mathrm{dd}, J=1.5$ and 1.5 $\mathrm{Hz}, 3 \mathrm{H}, 4^{\prime}-\mathrm{CH}_{3}$ ), 2.95 (dd, $J=17.1$ and $\left.3.3 \mathrm{~Hz}, 1 \mathrm{H}, 4-\mathrm{H}\right), 3.33$ (dd, $J=17.1$ and $\left.9.3 \mathrm{~Hz}, 1 \mathrm{H}, 4-\mathrm{H}\right), 3.96$ (dddd, $J=9.3,7.8,3.3$ and $2.4 \mathrm{~Hz}, 1 \mathrm{H}, 3 \mathrm{a}-\mathrm{H}), 5.86(\mathrm{~d}, J=7.8 \mathrm{~Hz}, 1 \mathrm{H}, 8 \mathrm{~b}-\mathrm{H}), 6.57$ (dq, $J=1.5$ and 1.5 Hz, 1H, 2'-H), 6.69 (br s, 1H, 5-H), 6.75 (d, J=8.4 Hz, 1H, 7-H), 7.28 (d, J = 8.4 Hz, 1H, 8-H), 7.32 (dq, $J=1.5$ and $\left.1.5 \mathrm{~Hz}, 1 \mathrm{H}, 3^{\prime}-\mathrm{H}\right), 7.55\left(\mathrm{~d}, J=2.4 \mathrm{~Hz}, 1 \mathrm{H}, 6{ }^{\prime}-\mathrm{H}\right){ }^{13}{ }^{13} \mathrm{C}$ NMR (acetone- $d_{6}$ ), $\delta 10.5,38.1,40.0$, 86.1, 102.1, 112.0, 114.1, 115.8, 127.9, 131.4, 135.5, 143.3, 145.8, 152.2, 160.0, 171.3, 171.4; HREIMS $m / z 314.0786[\mathrm{M}]^{+}\left(\mathrm{C}_{17} \mathrm{H}_{14} \mathrm{O}_{6}\right.$ requires 314.0790); EIMS m/z (rel. int.) $314[\mathrm{M}]^{+}$(19), 217 (21), 200 (14), 199 (9), 189 (17), 171 (45), 145 (26), 115 (19), 97 (100).

\subsubsection{3. (E)-7-Hydroxy-3-\{[(4-methyl-5-oxo-2,5-dihydrofuran-2-yl)oxy]methylene)-3,3a,4,8b- tetrahydro-2H-indeno[1,2-b]furan-2-one (7-hydroxy-GR24, 13)}

Each diastereomer of 7-MOMO-20 was deprotected under the same conditions as described in Section 4.3.5.1. 7-Hydroxy-GR24 (13a/c),14.3 mg from 37.3 mg of 7-MOMO-20a/c (43\% yield), as a white solid. Mp. $231-233{ }^{\circ} \mathrm{C} ;{ }^{1} \mathrm{H}$ NMR (acetone- $d_{6}$ ), $\delta 1.94$ (dd, $J=1.5$ and $1.5 \mathrm{~Hz}, 3 \mathrm{H}, 4{ }^{\prime}-\mathrm{CH}_{3}$ ), 2.95 (dd, $J=16.2$ and $3.0 \mathrm{~Hz}, 1 \mathrm{H}, 4-\mathrm{H}), 3.26(\mathrm{dd}, J=16.2$ and $9.0 \mathrm{~Hz}, 1 \mathrm{H}, 4-\mathrm{H}), 3.94$ (dddd, $J=9.0,7.8,3.0$ and $2.7 \mathrm{~Hz}, 1 \mathrm{H}, 3 \mathrm{a}-\mathrm{H}), 5.84(\mathrm{~d}, J=7.8 \mathrm{~Hz}, 1 \mathrm{H}, 8 \mathrm{~b}-\mathrm{H}), 6.53\left(\mathrm{dq}, J=1.5\right.$ and $\left.1.5 \mathrm{~Hz}, 1 \mathrm{H}, 2^{\prime}-\mathrm{H}\right), 6.79$ (dd, $J$ $=8.1$ and $2.4 \mathrm{~Hz}, 1 \mathrm{H}, 6-\mathrm{H}), 6.87(\mathrm{~d}, J=2.4 \mathrm{~Hz}, 1 \mathrm{H}, 8-\mathrm{H}), 7.03(\mathrm{~d}, J=8.1 \mathrm{~Hz}, 1 \mathrm{H}, 5-\mathrm{H}), 7.30(\mathrm{dq}, J=1.5$ and $\left.1.5 \mathrm{~Hz}, 1 \mathrm{H}, 3^{\prime}-\mathrm{H}\right), 7.53\left(\mathrm{~d}, J=2.7 \mathrm{~Hz}, 1 \mathrm{H}, 6^{\prime}-\mathrm{H}\right) ;{ }^{13} \mathrm{C}$ NMR (acetone- $d_{6}$ ), $\delta 10.5,37.0,40.1,86.3$, 102.2, 112.9, 113.5, 118.4, 126.7, 134.1, 135.5, 141.7, 143.2, 152.6, 157.8, 171.29, 171.33; HREIMS m/z 314.0784 [M] $]^{+}\left(\mathrm{C}_{17} \mathrm{H}_{14} \mathrm{O}_{6}\right.$ requires 314.0790); EIMS m/z (rel. int.) $314[\mathrm{M}]^{+}$(23), 217 (84), 200 (13), 199 (13), 171 (12), 161 (12), 145 (13), 115 (17), 97 (100). 7-Hydroxy-GR24 (13b/d), 16.3 mg from $35.7 \mathrm{mg}$ of 7-MOMO-20b/d (52\% yield), as a white solid. Mp. 192-194 ${ }^{\circ} \mathrm{C} ;{ }^{1} \mathrm{H}$ NMR (acetone- $\left.d_{6}\right), \delta 1.93(\mathrm{dd}, J=$ 1.5 and $\left.1.5 \mathrm{~Hz}, 3 \mathrm{H}, 4^{\prime}-\mathrm{CH}_{3}\right), 2.90(\mathrm{dd}, J=16.2$ and $3.3 \mathrm{~Hz}, 1 \mathrm{H}, 4-\mathrm{H}), 3.25(\mathrm{dd}, J=16.2$ and $9.0 \mathrm{~Hz}, 1 \mathrm{H}$, 
4-H), 3.94 (dddd, $J=9.0,7.8,3.3$ and $2.7 \mathrm{~Hz}, 1 \mathrm{H}, 3 \mathrm{a}-\mathrm{H}), 5.84(\mathrm{~d}, J=7.8 \mathrm{~Hz}, 1 \mathrm{H}, 8 \mathrm{~b}-\mathrm{H}), 6.52(\mathrm{dq}, J=1.5$ and $\left.1.5 \mathrm{~Hz}, 1 \mathrm{H}, 2^{\prime}-\mathrm{H}\right), 6.79(\mathrm{dd}, J=8.1$ and $2.4 \mathrm{~Hz}, 1 \mathrm{H}, 6-\mathrm{H}), 6.87(\mathrm{~d}, J=2.4 \mathrm{~Hz}, 1 \mathrm{H}, 8-\mathrm{H}), 7.03(\mathrm{~d}, J=$ 8.1 Hz, 1H, 5-H), 7.28 (dq, $J=1.5$ and $\left.1.5 \mathrm{~Hz}, 1 \mathrm{H}, 3^{\prime}-\mathrm{H}\right), 7.52\left(\mathrm{~d}, J=2.7 \mathrm{~Hz}, 1 \mathrm{H}, 6{ }^{\prime}-\mathrm{H}\right){ }^{13}{ }^{13} \mathrm{NMR}$ (acetone- $\left.d_{6}\right), \delta 10.5,37.2,40.1,86.3,102.1,112.8,113.8,118.4,126.7,134.1,135.5,141.6,143.2,152.4$, 157.8, 171.26, 171.32; HREIMS $m / z$ 314.0791 [M] $]^{+}\left(\mathrm{C}_{17} \mathrm{H}_{14} \mathrm{O}_{6}\right.$ requires 314.0790); EIMS $m / z$ (rel. int.) $314[\mathrm{M}]^{+}$(27), 217 (92), 200 (14), 199 (15), 171 (13), 161 (12), 145 (14), 115 (18), 97 (100).

\subsubsection{4. (E)-8-Hydroxy-3-\{[(4-methyl-5-oxo-2,5-dihydrofuran-2-yl)oxy]methylene)-3,3a,4,8b- tetrahydro-2H-indeno[1,2-b]furan-2-one (8-hydroxy-GR24, 14)}

Each diastereomer of 8-MOMO-20 was deprotected under the same conditions as described in Section 4.3.5.1. 8-Hydroxy-GR24 (14a/c), $12.1 \mathrm{mg}$ from $22.3 \mathrm{mg}$ of 8-MOMO-20a/c (62\% yield), as a white solid. Mp. $218-220{ }^{\circ} \mathrm{C} ;{ }^{1} \mathrm{H}$ NMR (acetone- $d_{6}$ ), $\delta 1.94\left(\mathrm{dd}, J=1.5\right.$ and $1.5 \mathrm{~Hz}, 3 \mathrm{H}, 4{ }^{\prime}-\mathrm{CH}_{3}$ ), 2.97 (dd, $J=16.8$ and $3.6 \mathrm{~Hz}, 1 \mathrm{H}, 4-\mathrm{H}), 3.35(\mathrm{dd}, J=16.8$ and $9.6 \mathrm{~Hz}, 1 \mathrm{H}, 4-\mathrm{H}), 3.93$ (dddd, $J=9.6,7.5,3.6$ and $2.4 \mathrm{~Hz}, 1 \mathrm{H}, 3 \mathrm{a}-\mathrm{H}), 6.00(\mathrm{~d}, J=7.5 \mathrm{~Hz}, 1 \mathrm{H}, 8 \mathrm{~b}-\mathrm{H}), 6.54(\mathrm{dq}, J=1.5$ and $1.5 \mathrm{~Hz}, 1 \mathrm{H}, 2$ '-H), $6.68(\mathrm{~d}, J$ $=7.5 \mathrm{~Hz}, 2 \mathrm{H}, 5-$ and $7-\mathrm{H}), 7.13(\mathrm{dd}, J=7.5$ and $7.5 \mathrm{~Hz}, 1 \mathrm{H}, 6-\mathrm{H}), 7.30\left(\mathrm{dq}, J=1.5\right.$ and $1.5 \mathrm{~Hz}, 1 \mathrm{H}, 3^{\prime}-$ $\mathrm{H}), 7.54\left(\mathrm{~d}, J=2.4 \mathrm{~Hz}, 1 \mathrm{H}, 6{ }^{\prime}-\mathrm{H}\right) ;{ }^{13} \mathrm{C}$ NMR (acetone- $\left.d_{6}\right), \delta 10.5,38.6,39.4,84.5,102.2,113.9,114.3$, 116.7, 126.7, 132.4, 135.5, 143.2, 146.3, 152.2, 156.3, 171.3, 171.5; HREIMS $m / z 314.0785$ [M] ${ }^{+}$ $\left(\mathrm{C}_{17} \mathrm{H}_{14} \mathrm{O}_{6}\right.$ requires 314.0790); EIMS m/z (rel. int.) $314[\mathrm{M}]^{+}$(14), $296\left[\mathrm{M}-\mathrm{H}_{2} \mathrm{O}\right]^{+}$(9), 217 (23), 200 (14), 199 (15), 171 (16), 145 (10), 115 (19), 97 (100). 8-Hydroxy-GR24 (14b/d), 15.3 mg from 35.6 mg of 8MOMO-20b/d (49\% yield), as a white solid. Mp. $219-222{ }^{\circ} \mathrm{C} ;{ }^{1} \mathrm{H}$ NMR (acetone- $\left.d_{6}\right), \delta 1.98(\mathrm{dd}, J=1.5$ and $\left.1.5 \mathrm{~Hz}, 3 \mathrm{H}, 4^{\prime}-\mathrm{CH}_{3}\right), 2.96(\mathrm{dd}, J=17.1$ and $3.9 \mathrm{~Hz}, 1 \mathrm{H}, 4-\mathrm{H}), 3.38(\mathrm{dd}, J=17.1$ and $9.6 \mathrm{~Hz}, 1 \mathrm{H}, 4-$ H), 3.97 (dddd, $J=9.6,7.8,3.9$ and $2.4 \mathrm{~Hz}, 1 \mathrm{H}, 3 \mathrm{a}-\mathrm{H}), 6.03(\mathrm{~d}, J=7.8 \mathrm{~Hz}, 1 \mathrm{H}, 8 \mathrm{~b}-\mathrm{H}), 6.57$ (dq, $J=1.5$ and $\left.1.5 \mathrm{~Hz}, 1 \mathrm{H}, 2^{\prime}-\mathrm{H}\right), 6.72(\mathrm{~d}, J=7.5 \mathrm{~Hz}, 2 \mathrm{H}, 5-$ and $7-\mathrm{H}), 7.16(\mathrm{dd}, J=7.5$ and $7.5 \mathrm{~Hz}, 1 \mathrm{H}, 6-\mathrm{H}), 7.32$ (dq, $J=1.5$ and $\left.1.5 \mathrm{~Hz}, 1 \mathrm{H}, 3^{\prime}-\mathrm{H}\right), 7.57$ (d, $\left.J=2.4 \mathrm{~Hz}, 1 \mathrm{H}, 6^{\prime}-\mathrm{H}\right) ;{ }^{13} \mathrm{C}$ NMR (acetone- $d_{6}$ ), $\delta 10.6,38.8$, $39.4,84.5,102.1,114.2,114.3,116.8,126.6,132.4,135.5,143.3,146.3,152.1,156.3,171.3,171.5$; 
HREIMS m/z $314.0794[\mathrm{M}]^{+}\left(\mathrm{C}_{17} \mathrm{H}_{14} \mathrm{O}_{6}\right.$ requires 314.0790); EIMS m/z (rel. int.) $314[\mathrm{M}]^{+}(14), 296[\mathrm{M}-$ $\left.\mathrm{H}_{2} \mathrm{O}\right]^{+}(10), 217$ (21), 200 (14), 199 (16), 171 (15), 145 (9), 115 (17), 97 (100).

\subsubsection{Optical resolution of hydroxyl GR24s}

Each of the racemates $11 a / c, 11 b / d, 12 a / c, 12 b / d, 13 a / c$ and $14 a / c$ was optically resolved by chiral HPLC on a CHIRALPAK IC $(250 \times 10 \mathrm{~mm}, 5 \mu \mathrm{m})$ with hexane/EtOH $(1: 1)$ as the mobile phase at a flow rate of $1.2 \mathrm{~mL} / \mathrm{min}$. The mixture of hexane/EtOH (7:3) was used as the mobile phase for optical resolution of $\mathbf{1 3 b} / \mathbf{d}$. Optical resolution of $\mathbf{1 4 b} / \mathbf{d}$ was performed using a CHIRALPAK IA $(250 \times 10 \mathrm{~mm}$, 5 um) column with hexane/EtOH (1:1) as the mobile phase. The column effluent was monitored at 235 $\mathrm{nm}$ to give the following enantiomers: 11a (at $28 \mathrm{~min}$ ) and 11c (at $42 \mathrm{~min}$ ), 11d (at $20 \mathrm{~min}$ ) and $\mathbf{1 1 b}$ (at $25 \mathrm{~min}$ ), 12a (at $33 \mathrm{~min}$ ) and $\mathbf{1 2 c}($ at $41 \mathrm{~min}), \mathbf{1 2 d}($ at $21 \mathrm{~min})$ and $\mathbf{1 2 b}$ (at $28 \mathrm{~min}$ ), 13a (at $28 \mathrm{~min}$ ) and $\mathbf{1 3 c}($ at $42 \mathrm{~min}$ ), $\mathbf{1 3 d}$ (at $43 \mathrm{~min}$ ) and $\mathbf{1 3 b}$ (at $61 \mathrm{~min}$ ), $\mathbf{1 4 a}$ (at $32 \mathrm{~min}$ ) and $\mathbf{1 4 c}$ (at $40 \mathrm{~min}$ ), and $\mathbf{1 4 b}$ (at 23 $\min )$ and 14d (at $33 \mathrm{~min}) . \mathrm{CD}, \lambda_{\mathrm{ext}},(\Delta \varepsilon)$ : 11a $(c$ 0.000039), 264 (-1.1), $229(+40.8), 203$ (-29.6) nm; 11b (c 0.000041), $233(+5.5), 205(-9.1) \mathrm{nm} ; 11 \mathrm{c}(c 0.000044), 265(+1.1), 230(-38.8), 204(+29.7) \mathrm{nm} ; \mathbf{1 1 d}$ (c 0.000032), 232 (-7.2), $205(+14.0) \mathrm{nm}$; 12a (c 0.000035), $269(-1.1), 235(+39.1), 206(-9.1) \mathrm{nm} ; \mathbf{1 2 b}$ (c 0.000053), 237 (+16.5), 218 (-6.6), $211(-4.1), 206$ (-8.7) nm; 12c (c 0.000047), 265 (+1.0), 235 (-38.0), 205 (9.3) nm; 12d (c 0.000053), 238 (-14.2), $219(+6.1), 212(+4.6), 206(+8.7) \mathrm{nm} ; \mathbf{1 3 a}(c$ 0.000044), $285(+3.1), 260(-2.0), 228(+22.2), 209(+6.4), 201(+21.6) \mathrm{nm} ; \mathbf{1 3 b}(c 0.000041), 289(+2.3), 269(+0.8)$, $241(+4.5), 218(-1.7), 201(+26.0) \mathrm{nm} ; 13 \mathrm{c}(c 0.000041), 284$ (-4.5), $262(+0.3), 227(-24.9), 210(-7.7)$, $202(-23.0) \mathrm{nm} ; \mathbf{1 3 d}(c$ 0.000043), $282(-3.6), 266(-2.4), 238(-6.3), 219(-0.1), 201(-25.8) \mathrm{nm} ; \mathbf{1 4 a}(c$ 0.000053), 280 (+1.9), 260 (-0.3), 229 (+31.1), $204(-16.2) \mathrm{nm} ; \mathbf{1 4 b}(c 0.000056), 273$ (+2.7), 260 (+2.2), 231 (+9.5), 206 (-8.2) nm; 14c (c 0.000059), 280 (-1.8), 262 (+0.2), $228(-29.0), 204(+17.5) \mathrm{nm} ; \mathbf{1 4 d}(c$ 0.000056), $272(-2.8), 262(-2.2), 231(-9.4), 205(+8.1) \mathrm{nm}$.

Perfect mirror images in CD curves of the respective enantiomer were recorded (Fig. 4). 


\subsection{Feeding to sorghum and germination assay}

\subsubsection{Plant materials}

Seeds of high sorgomol-producing sorghum (Sorghum bicolor) were supplied by Kaneko Seeds, Gunma, Japan. Striga gesnerioides and S. hermonthica seeds, collected from mature plants parasitizing cowpea and sorghum, respectively, were kindly supplied by Dr. Satoru Muranaka, International Institute of Tropical Agriculture, Nigeria, and Prof. Abdel Gabar Babiker, Sudan University of Science and Technology, respectively.

\subsubsection{Feeding of GR24 stereoisomers and extraction of the metabolites}

Feeding procedures were almost the same as those employed for administration of 5-deoxystrigol to sorghum as described previously. ${ }^{18}$ Briefly, each sorghum seedling was grown hydroponically with 40\% Long Ashton nutrient solution for 2 weeks or modified Hoagland's solution (1/2-strength for 4 days, 1/2-strength with $50 \mu \mathrm{M}$ phosphate for 3 days, and 1/4-strength with $25 \mu \mathrm{M}$ phosphate for 2 days and without phosphate for a day) in a falcon tube $(50 \mathrm{~mL})$, and seedlings were then transferred to tap water. After 2-3 days, GR24 [racemic and diastereomeric mixture (5) at $4 \mu \mathrm{M}$, each stereoisomer (5a-d) at $2 \mu \mathrm{M}$ in tap water] was administered to the seedlings for $24 \mathrm{~h}$. The sorghum roots were washed with tap water quickly and stored at $-80{ }^{\circ} \mathrm{C}$ for a few hours. The frozen roots $(4-8 \mathrm{~g})$ were homogenized in a mixture of acetone/EtOH (1:1) $(25 \mathrm{~mL})$ and the solution was concentrated in vacuo after filtration. The residual solution was added to brine $(10 \mathrm{~mL})$ and then extracted with a mixture of hexane/EtOAc $(1: 1)(6 \mathrm{~mL} \times 4)$. The organic layers pooled and concentrated in vacuo. The residue was applied onto a silica gel column $(1.5 \mathrm{~mL})$ with hexane and washed with hexane $/ \mathrm{CH}_{2} \mathrm{Cl}_{2}(1: 4)(10 \mathrm{~mL})$. GR24 and its metabolites were eluted with $\mathrm{CH}_{2} \mathrm{Cl}_{2} / \mathrm{EtOAc}(3: 1)(10 \mathrm{~mL})$ and the eluent was removed in vacuo. The residue was dissolved in MeCN $(120 \mu \mathrm{L})$, filtered with a PTFE filter $(0.2 \mu \mathrm{m})$ and analyzed using LC-MS. The sorghum root exudates were collected using a reverse phased-column (NOBIAS RP-EX1, $1.3 \mathrm{~mL}$, Hitachi High-Technologies Corp., Tokyo, Japan) from the aquaculture filtrate and eluted with $\mathrm{EtOH} / \mathrm{H}_{2} \mathrm{O}(4: 1)$. 
Alternatively, the exudates were extracted with liquid-liquid partition using EtOAc, which effected a recovery efficiency comparable to the reverse-phased column. The organic solution containing the root exudates were concentrated in vacuo and the residue was applied onto a silica gel column $(0.5 \mathrm{~mL})$. The root exudates were eluted with $\mathrm{CH}_{2} \mathrm{Cl}_{2} / \mathrm{EtOAc}$ (3:1) and the eluent was removed in vacuo. The residue was dissolved in MeCN (120 $\mu \mathrm{L})$ and filtered with a PTFE filter for LC-MS analysis.

\subsubsection{Acetylation of GR24 metabolites}

The root exudates of sorghum previously fed with GR24 (4 $\mu \mathrm{M}$ of 5) were collected with NOBIAS and eluted with EtOH/ $\mathrm{H}_{2} \mathrm{O}(4: 1)$. The eluent was concentrated in vacuo at $35{ }^{\circ} \mathrm{C}$ and the residue was dissolved in acetic anhydride $\left(\mathrm{Ac}_{2} \mathrm{O}\right)(0.3 \mathrm{~mL})$ and a small amount of trichloroacetic acid (TCA) was added to the solution. The mixture was stirred for $22 \mathrm{~h}$ at $\mathrm{RT}$ and brine $(3 \mathrm{~mL})$ was then added. The solution was extracted with EtOAc $(3 \mathrm{~mL} \times 3)$ and the organic layers were pooled, toluene was added and the solution was concentrated at $40{ }^{\circ} \mathrm{C}$. The residue was roughly purified with silica gel $\mathrm{CC}$ using $\mathrm{CH}_{2} \mathrm{Cl}_{2} / \mathrm{EtOAc}$ (3:1) before LC-MS analysis. As a control experiment, the methyl ester of abscisic acid $(1.3 \mathrm{mg})$ was dissolved in $\mathrm{Ac}_{2} \mathrm{O}(0.5 \mathrm{~mL})$ and a small amount of TCA was added to the solution. The mixture was stirred for $22 \mathrm{~h}$ at RT in the dark and the reaction was quenched by addition of brine (10 $\mathrm{mL}$ ). The resulting mixture was extracted with EtOAc and the products in the organic solution were analyzed by TLC on silica gel with a solvent of hexane/EtOAc (1:1). The TLC plate was examines under UV light at $254 \mathrm{~nm}$.

\subsubsection{LC-MS analysis of GR24 metabolites}

LC-MS/MS analyses were performed using an LC-MS/MS system (Waters, Milford, MA) consisting of an Acquity Ultra Performance liquid chromatography (UPLC) and an Acquity quadruple tandem mass spectrometer (TQ Detector). Data acquisition and analyses were performed using MassLynx 4.1 software (Waters, Milford, MA). UPLC analytical conditions were as follows: column, $100 \times 2.0 \mathrm{~mm}$, 
$2 \mu \mathrm{m}$, YMC-UltraHT Hydrosphere C18 (YMC Co., Ltd., Kyoto, Japan), $30{ }^{\circ} \mathrm{C}$; solvent, $\mathrm{MeOH} / \mathrm{H}_{2} \mathrm{O}$ (7:13 for $2 \mathrm{~min}, 7: 13$ to 9:11 over $15 \mathrm{~min}, 9: 11$ to 20:0 over $5 \mathrm{~min}$, linear gradient); flow rate, 0.2 $\mathrm{mL} / \mathrm{min}$. The mass spectrometer was operated in positive eletrospray ionization (ESI) mode with the capillary voltage at $3 \mathrm{kV}$, the source temperature at $120^{\circ} \mathrm{C}$, and the desolvation gas temperature at 350 ${ }^{\circ} \mathrm{C}$. The nebulizer and desolvation $\mathrm{N}_{2}$ gas flows were 50 and $550 \mathrm{~L} / \mathrm{h}$, respectively. The fragmentation was performed by collision-induced dissociation (CID) with argon at $0.1 \mathrm{~mL} / \mathrm{min}$. The MRM transitions were set according to the mass spectra obtained for the standards: at $m / z 299.1>97.0$ and $299.1>185.1$

for GR24, $m / z 315.1>97.0,315.1>155.1$, and 315.1 > 173.1, for hydroxyl GR24s, a corn voltage of 30 $\mathrm{V}$, and a collision energy of $20 \mathrm{eV}$. To confirm the fragment pattern of authentic compounds and GR24 metabolites, a daughter ion scan was performed for precursor ions at $m / z 299,315$ and 357 over the range of 50-400 $\mathrm{m} / \mathrm{z}$, with a cone voltage of $30-35 \mathrm{~V}$ and a collision energy of $10 \mathrm{eV}$.

\subsubsection{Germination assay}

The germination assay was conducted as reported previously. ${ }^{24}$ Seeds of $S$. gesnerioides and $S$. hermonthica were surface sterilized by immersion in $0.75 \%(\mathrm{w} / \mathrm{v}) \mathrm{NaOCl}$, containing few drops of Tween 20, and sonication for $3 \mathrm{~min}$ in an ultrasonic cleaner. After having been rinsed with distilled water and surface-dried in a laminar hood, the seeds were pretreated (conditioned) for $8-12$ days at $28{ }^{\circ} \mathrm{C}$ on $8 \mathrm{~mm}$ glass fiber filter paper disks ( $c a .50$ seeds each) placed on distilled water-saturated filter paper. Aliquots $(20 \mu \mathrm{L})$ of dilution series of the test solutions were assayed by applying them to the conditioned Striga seeds on the $8 \mathrm{~mm}$ discs. The treated seeds were incubated at the same temperature as conditioning and were microscopically examined for germination (radical protrusion) after $24 \mathrm{~h}$ in case of $S$. hermonthica and $48 \mathrm{~h}$ in case of $S$. gesnerioides.

\section{Acknowledgements}


The authors are grateful to Prof. Abdel Gabar Babiker, Sudan University of Science and

Technology, for critical reading of the manuscript. This work was supported, in part, by grants from JST/JICA, Science and Technology Research Partnership for Sustainable Development (SATREPS) and Grants-in-Aid for Scientific Research from the Ministry of Education, Culture, Sports, Science and Technology of Japan (Nos. 11J1777, 24658111, 25292065 and 15H05248).

\section{References}

1. Parker C. Pest Manag. Sci. 2009, 65, 453.

2. Cook, C. E.; Whichard, L. P.; Wall, M. E.; Egley, G. H.; Coggon, P.; Luhan P.A.; McPhail, A.T. J. Amer. Chem. Soc. 1972, 94, 6198.

3. Ueno, K.; Sugimoto, Y.; Zwanenburg, B. Phytochem. Rev. in press, DOI 10.1007/s11101-014-9380-2.

4. Ruyter-Spira, C.; Al-Babili, S.; van der Krol, S.; Bouwmeester, H. Trends Plant Sci. 2013, 18, 72.

5. Matusova, R.; Rani, K.; Verstappen, F. W. A.; Franssen, M. C. R.; Beale, M. H.; Bouwmeester, H. J. Plant Physiol. 2005, 139, 920.

6. Seto, Y.; Sado, A.; Asami, K.; Hanada, A.; Umehara, M.; Akiyama, K.; Yamaguchi, S. Proc. Natl. Acad. Sci. USA. 2014, 111, 1640.

7. Alder, A.; Jamil, M.; Marzorati, M.; Bruno, M.; Vermathen, M.; Bigler, P.; Ghisla, S.; Bouwmeester, H.; Beyer, P.; Al-Babili, S. Science. 2012, 335, 1348.

8. Zhang, Y.; van Dijk, A. D.; Scaffidi, A.; Flematti, G. R.; Hofmann, M.; Charnikhova, T.; Verstappen, F.; Hepworth, J.; van der Krol, S.; Leyser, O.; Smith, S. M.; Zwanenburg, B.; Al-Babili, S.; RuyterSpira, C.; Bouwmeester, H. J. Nat. Chem. Biol. 2014, 10, 1028.

9. Johnson, A. W.; Gowda, G.; Hassanali, A.; Knox, J.; Monaco, S.; Razavi, Z.; Rosebery, G. J. Chem. Soc. Perkin Trans 1. 1981, 1734.

10. Kondo, Y.; Tadokoro, E.; Matsuura, M.; Iwasaki, K.; Sugimoto, Y.; Miyake, H.; Takikawa, H.; Sasaki, M. Biosci. Biotechnol. Biochem. 2007, 71, 2781. 
11. Zwanenburg, B.; Pospísil, T. Mol. Plant. 2013, 6, 38.

12. Müller, S.; Hauck, C.; Schildknecht, H. J. Plant Growth Regul. 1992, 11, 77.

13. Ueno, K.; Nomura, S.; Muranaka, S.; Mizutani, M.; Takikawa, H.; Sugimoto, Y. J. Agric. Food Chem. 2011, 59, 10485.

14. Ueno, K.; Fujiwara, M.; Nomura, S.; Mizutani, M.; Sasaki, M.; Takikawa, H.; Sugimoto, Y. J. Agric. Food Chem. 2011, 59, 9226.

15. Nomura, S.; Nakashima, H.; Mizutani, M.; Takikawa, H.; Sugimoto, Y. Plant Cell Rep. 2013, 32, 829.

16. Akiyama, K.; Ogasawara, S.; Ito, S.; Hayashi, H. Plant Cell Physiol. 2010, 51, 1104.

17. Chen, V. X.; Boyer, F.D.; Rameau, C.; Retailleau, P.; Vors, J. P.; Beau, J. M. Chem. Eur. J. 2010, 16, 13941.

18. Motonami, N.; Ueno, K.; Nakashima, H.; Nomura, S.; Mizutani, M.; Takikawa, H.; Sugimoto, Y. Phytochemistry. 2013, 93, 41.

19. Malik, H.; Kohlen, W.; Jamil, M.; Rutjes, F. P. J. T.; Zwanenburg, B. Org. Biomol. Chem. 2011, 9 , 2286.

20. Mangnus, E. M.; Dommerholt, F. J.; De Jong, R. L. P.; Zwanenburg, B. J. Agric. Food Chem. 1992, $40,1230$.

21. Zwanenburg, B.; Mwakaboko, A. S.; Reizelman, A.; Anilkumar, G.; Sethumadhavan, D. Pest Manag. Sci. 2009, 65, 478 .

22. Schuler, M. A. Crit. Rev. Plant Sci. 1996, 15, 235

23. Robineau, T.; Batard, Y.; Nedelkina, S.; Cabello-Hurtado, F.; LeRet, M.; Sorokine, O.; Didierjean, L.; Werck-Reichhart, D. Plant Physiol. 1998, 118, 1049.

24. Sugimoto, Y.; Ali, A. M.; Yabuta, S.; Kinoshita, H.; Inanaga, S.; Itai, A. Physiol. Plant. 2003, 19, 137. 
Figure legends

Figure 1. Structures of strigolactones and carlactone (2), a common precursor of natural strigolactones.

Figure 2. Sorghum converted GR24 into hydroxylated products. TIC chromatograms of the root extracts (A), the root exudates (B) and the acetylated root exudates (C). Ion intensities of the chromatograms were normalized by the intensity of the largest peak in (B) (8.79e6) that was residual GR24 in aquaculture filtrate. The MRM chromatograms of the transition at $m / z 315>97$ were depicted in the boxes, and the $y$ axes of the chromatograms were normalized by the intensity of the peak iii in (B) (5.38e4). MS/MS fragmentation patterns of GR24 metabolites (D). Proton adduct ions at $m / z$ 299, 315 and 357 in GR24, hydroxylated GR24 (compound 9 and peaks i-iv) and acetylated metabolites, respectively, were selected as a precursor ion in the daughter scan mode with the collision energy at $10 \mathrm{eV}$.

Figure 3. Synthesis of hydroxylated GR24 analogues (A). The scheme is depicted with common structures of regioisomers. Reagents and conditions: i) $\mathrm{MOMCl}$, DIPEA in $\mathrm{CH}_{2} \mathrm{Cl}_{2}, \mathrm{RT}, 74-92 \%$; ii) $\mathrm{ICH}_{2} \mathrm{CO}_{2} \mathrm{Et}$, LDA in THF, $-78^{\circ} \mathrm{C}, 53-72 \%$; iii) $1 \mathrm{M} \mathrm{NaOH}$ aq., reflux; iv) NBH, in $1 \mathrm{M} \mathrm{NaOH}$ aq., RT; v) $1 \mathrm{M} \mathrm{HCl}$, RT, $13-27 \%$ (3 steps); vi) $\mathrm{HCO}_{2} \mathrm{CH}_{3}, \mathrm{NaH}$ in $\mathrm{Et}_{2} \mathrm{O}$, RT; vii) compound 19, $\mathrm{K}_{2} \mathrm{CO}_{3}$ in $\mathrm{DME}$, RT, 33-53\% (2 steps); and viii) TFA in $\mathrm{CH}_{2} \mathrm{Cl}_{2}, \mathrm{RT}, 30-72 \%$. Absolute configuration of stereoisomers of GR24 analogues (B).

Figure 4. CD spectra of hydroxylated GR24 stereoisomers.

Figure 5. LC-MS analyses of hydroxylated GR24 metabolites of sorghum by MRM channel at $m / z 315>$ 97. Authentic hydroxyl GR24 diastereomers (compounds 9-14) (A). Metabolites of racemic and diastereomeric mixture of GR24 by sorghum (B). Metabolites of each of GR24 stereoisomers, 5a, 5b, 5c 
and 5d (see Fig. 3B) and mixtures of authentic hydroxyl GR24 diastereomers (C). Intensities of chromatograms for the metabolites of $\mathbf{5 a}, \mathbf{5 b}, \mathbf{5 c}$, and $\mathbf{5 d}$ were $1.03 \mathrm{e} 4,1.19 \mathrm{e} 5,5.73 \mathrm{e} 3$, and $1.21 \mathrm{e} 4$, respectively. Peak areas of GR24 metabolites hydroxylated at C-4, C-7 or C-8 (D).

Figure 6. Germination-inducing activities of GR24 (5) and its hydroxylated analogues, trans-4-HO-GR24 (9), cis-4-HO-GR24 (10), 5-HO-GR24 (11), 6-HO-GR24 (12), 7-HO-GR24 (13) and 8-HO-GR24 (14), toward Striga hermonthica and S. gesnerioides. Configurations of stereoisomers of GR24 analogues are shown in Fig 3B. Data represent the mean of 5 discs. In the positive control experiment toward $S$. gesnerioides, germination achieved by 4 at $0.1 \mu \mathrm{M}$ was $48.2 \pm 2.8 \%$. 


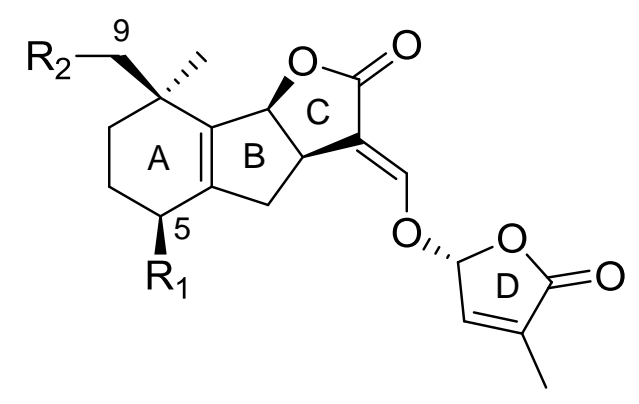

Strigol (1): $\mathrm{R}_{1}=\mathrm{OH}, \mathrm{R}_{2}=\mathrm{H}$ Sorgomol (7): $\mathrm{R}_{1}=\mathrm{H}, \mathrm{R}_{2}=\mathrm{OH}$ 5-Deoxystrigol (8): $\mathrm{R}_{1}=\mathrm{R}_{2}=\mathrm{H}$

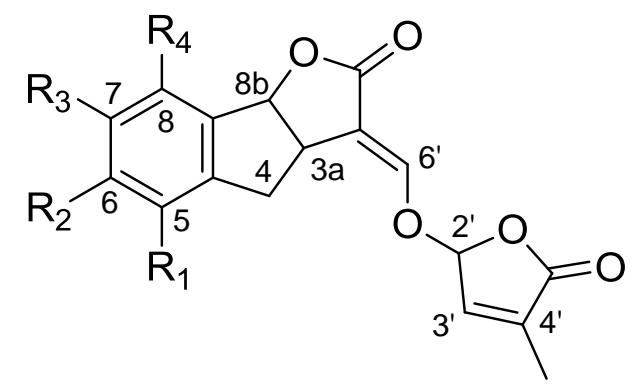

GR24 (5): $R_{1}=R_{2}=R_{3}=R_{4}=H$ 5-HO-GR24 (11): $\mathrm{R}_{1}=\mathrm{OH}, \mathrm{R}_{2}=\mathrm{R}_{3}=\mathrm{R}_{4}=\mathrm{H}$ 6-HO-GR24 (12): $\mathrm{R}_{1}=\mathrm{H}, \mathrm{R}_{2}=\mathrm{OH}, \mathrm{R}_{3}=\mathrm{R}_{4}=\mathrm{H}$ 7-HO-GR24 (13): $\mathrm{R}_{1}=\mathrm{R}_{2}=\mathrm{H}, \mathrm{R}_{3}=\mathrm{OH}, \mathrm{R}_{4}=\mathrm{H}$ 8-HO-GR24 (14): $R_{1}=R_{2}=R_{3}=H, R_{4}=O H$

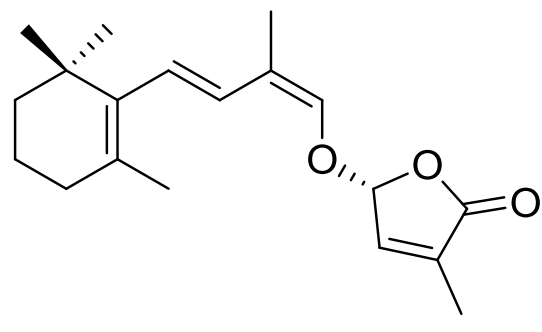

Carlactone (2)

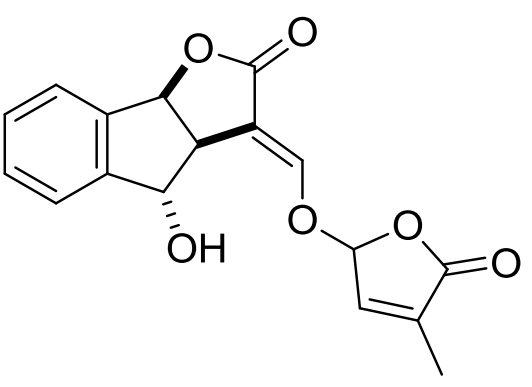

trans-4-HO-GR24 (9)

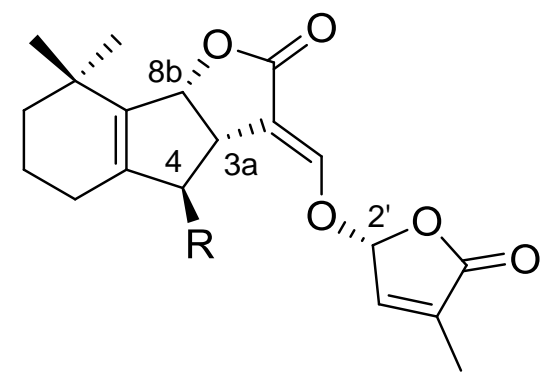

4-Deoxyorobanchol (3): $\mathrm{R}=\mathrm{H}$ Orobanchol (4): $\mathrm{R}=\mathrm{OH}$ Alectrol (6): $\mathrm{R}=\mathrm{OAc}$

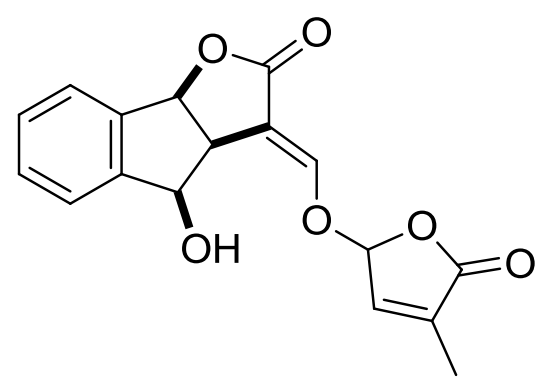

cis-4-HO-GR24 (10) 


\section{Fig. 2}

D
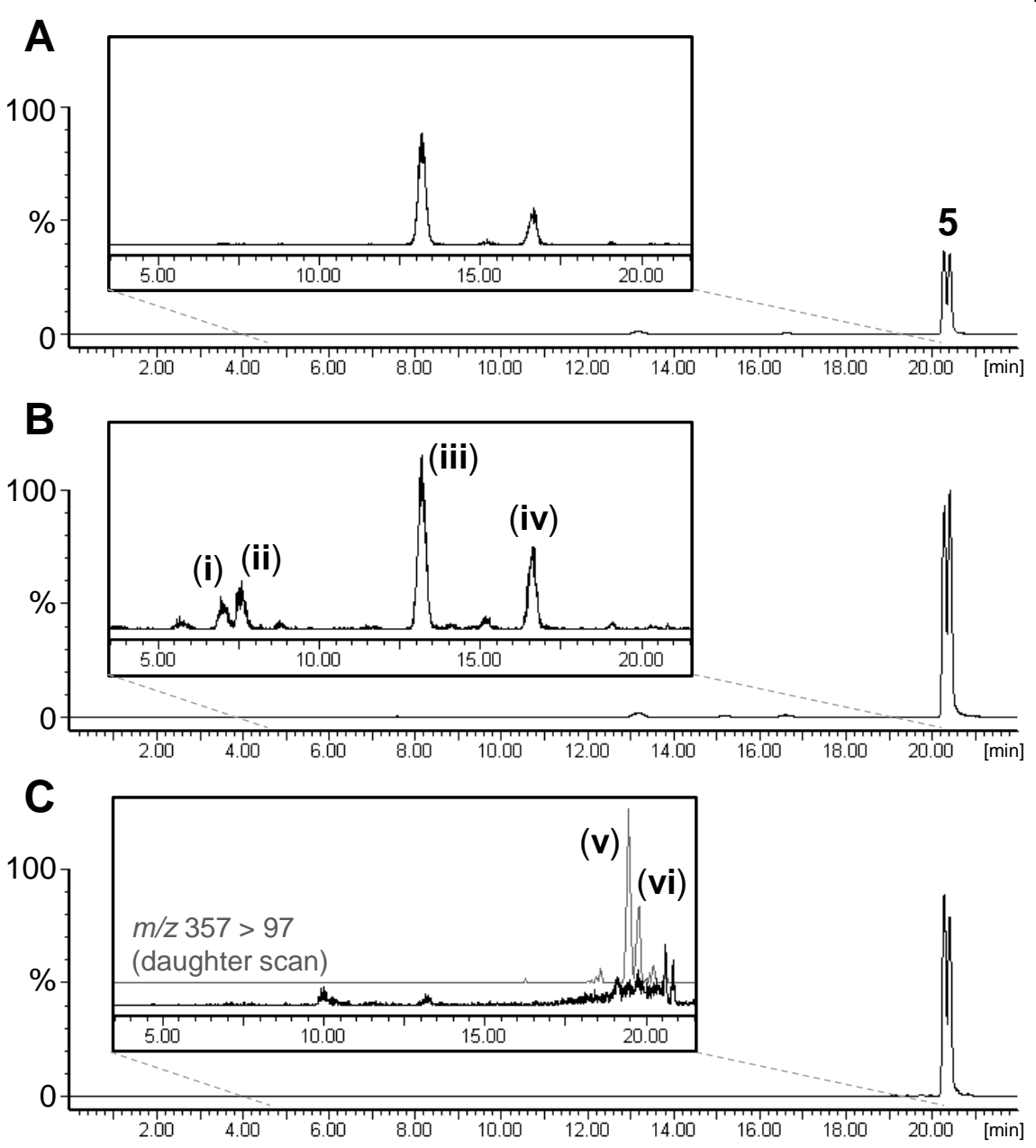

\section{GR24 (5) $(m / z$ 299)}

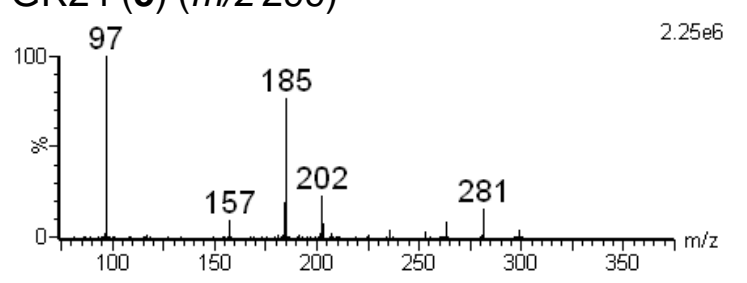
trans-4-HO-GR24 (9) (m/z 315)

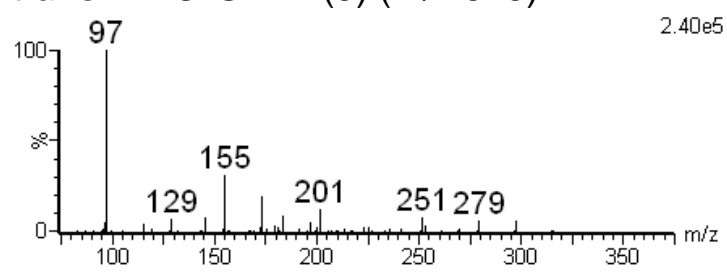

(i) $7.0 \mathrm{~min}(\mathrm{~m} / \mathrm{z} 315)$

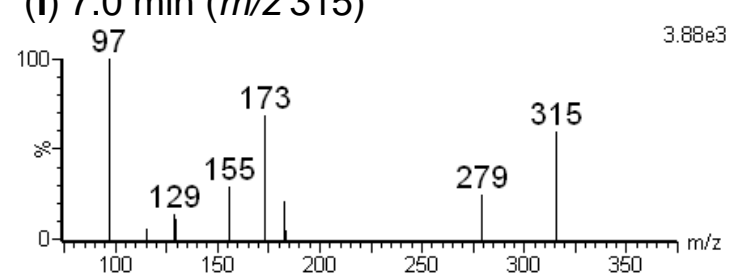

(ii) $7.6 \mathrm{~min}(\mathrm{~m} / \mathrm{z} 315)$

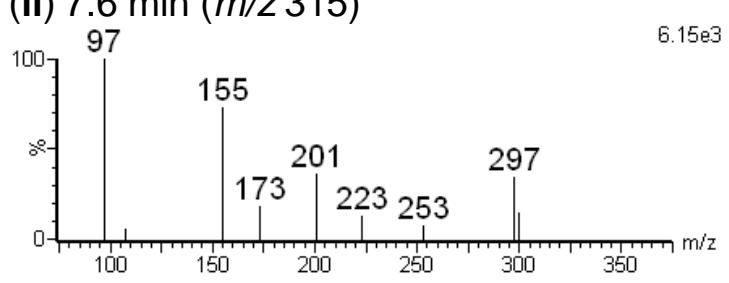

(iii) $13.2 \mathrm{~min}(\mathrm{~m} / \mathrm{z} 315)$

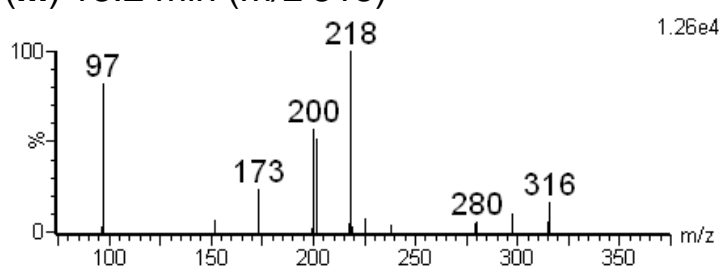

(iv) $16.6 \mathrm{~min}(\mathrm{~m} / \mathrm{z} 315)$

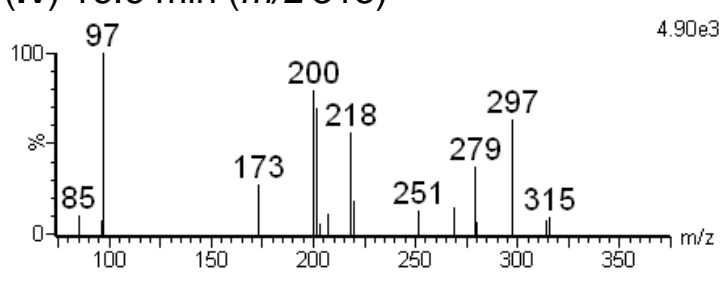

(v) $19.4 \mathrm{~min}(\mathrm{~m} / \mathrm{z} 357)$

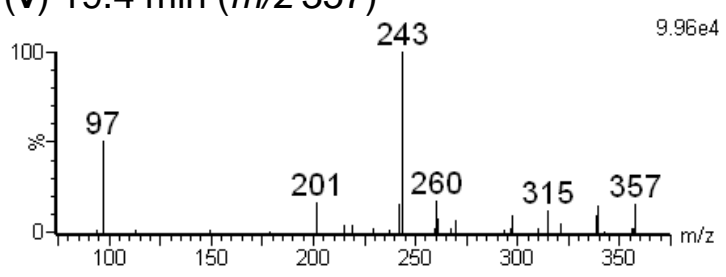

(vi) $19.8 \mathrm{~min}(\mathrm{~m} / \mathrm{z} 357)$

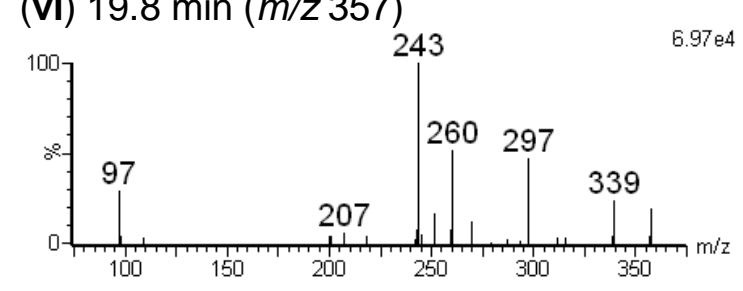




\section{Fig. 3}

A
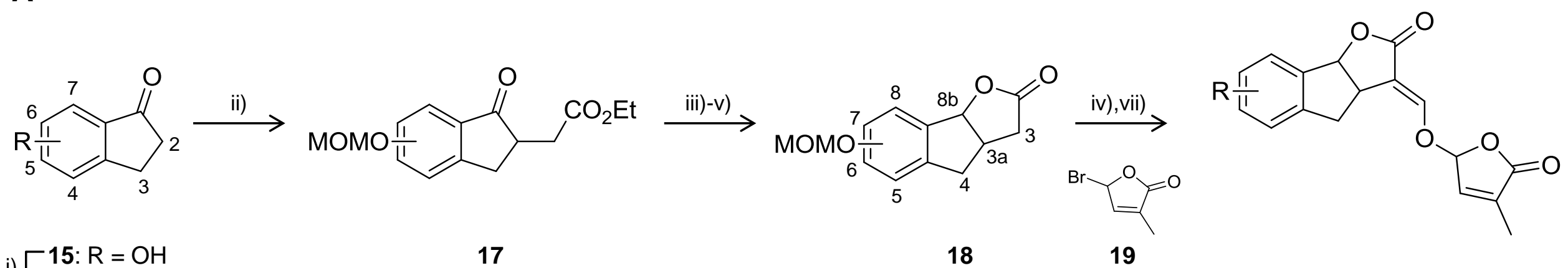

15: $\mathrm{R}=\mathrm{OH}$
$\rightarrow 16: \mathrm{R}=\mathrm{OMOM}$

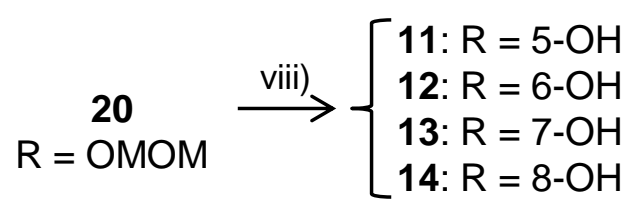

B
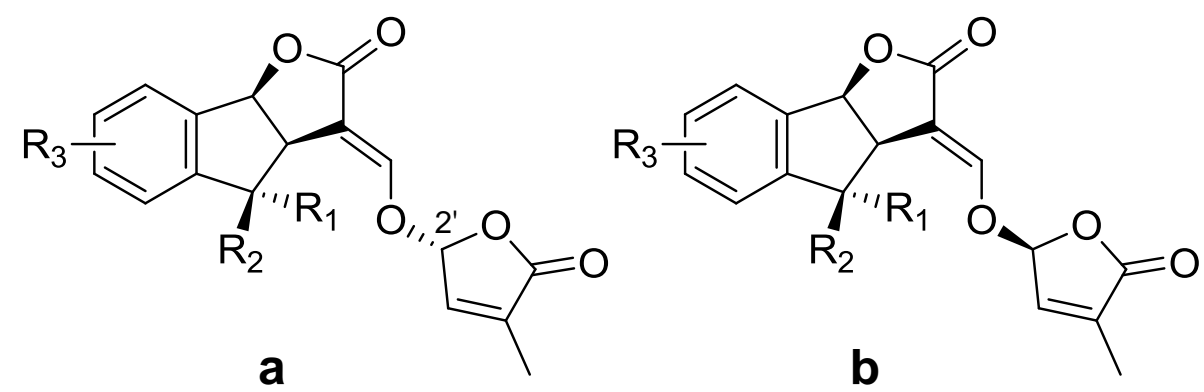

2'-epi-

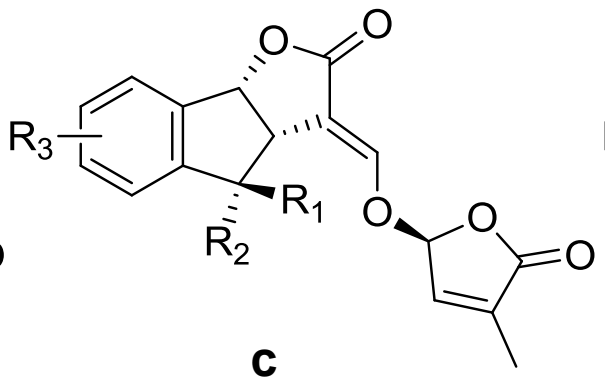

ent-

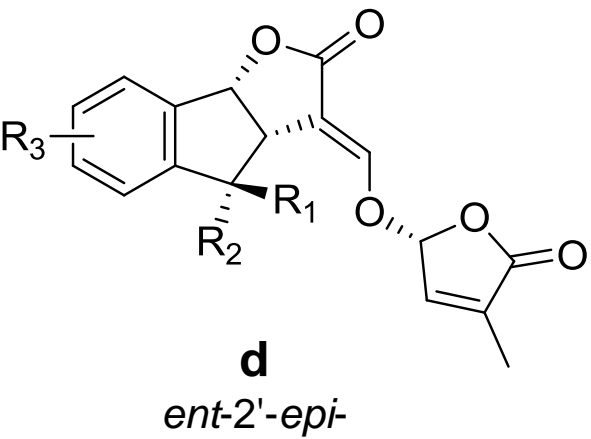

ent-2'-epi-

5, $R_{1}=R_{2}=R_{3}=H ; 9, R_{1}=O H, R_{2}=R_{3}=H ; 10, R_{1}=H, R_{2}=O H, R_{3}=H ; 11-14, R_{1}=R_{2}=H, R_{3}=O H$ 

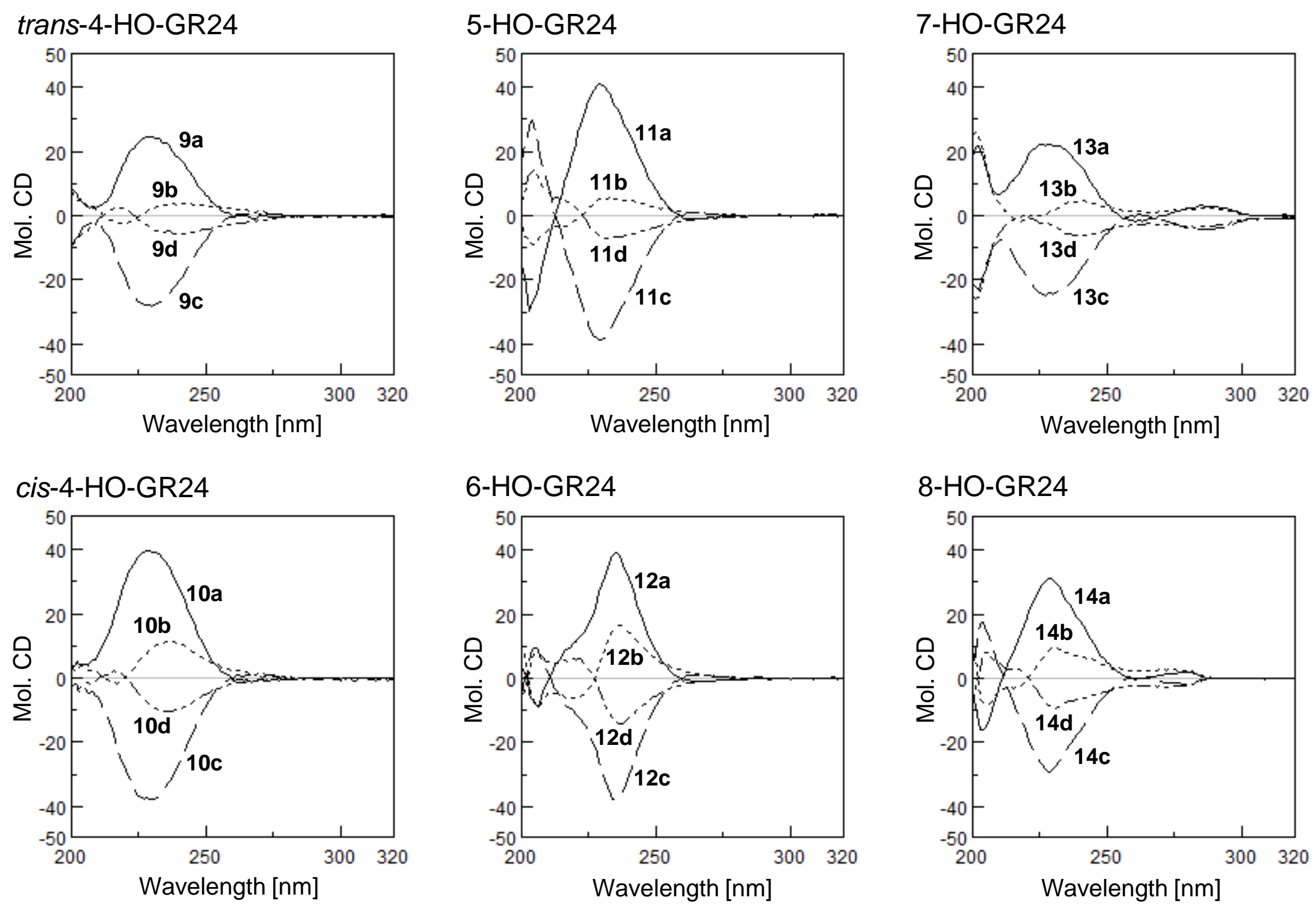
Fig. 5

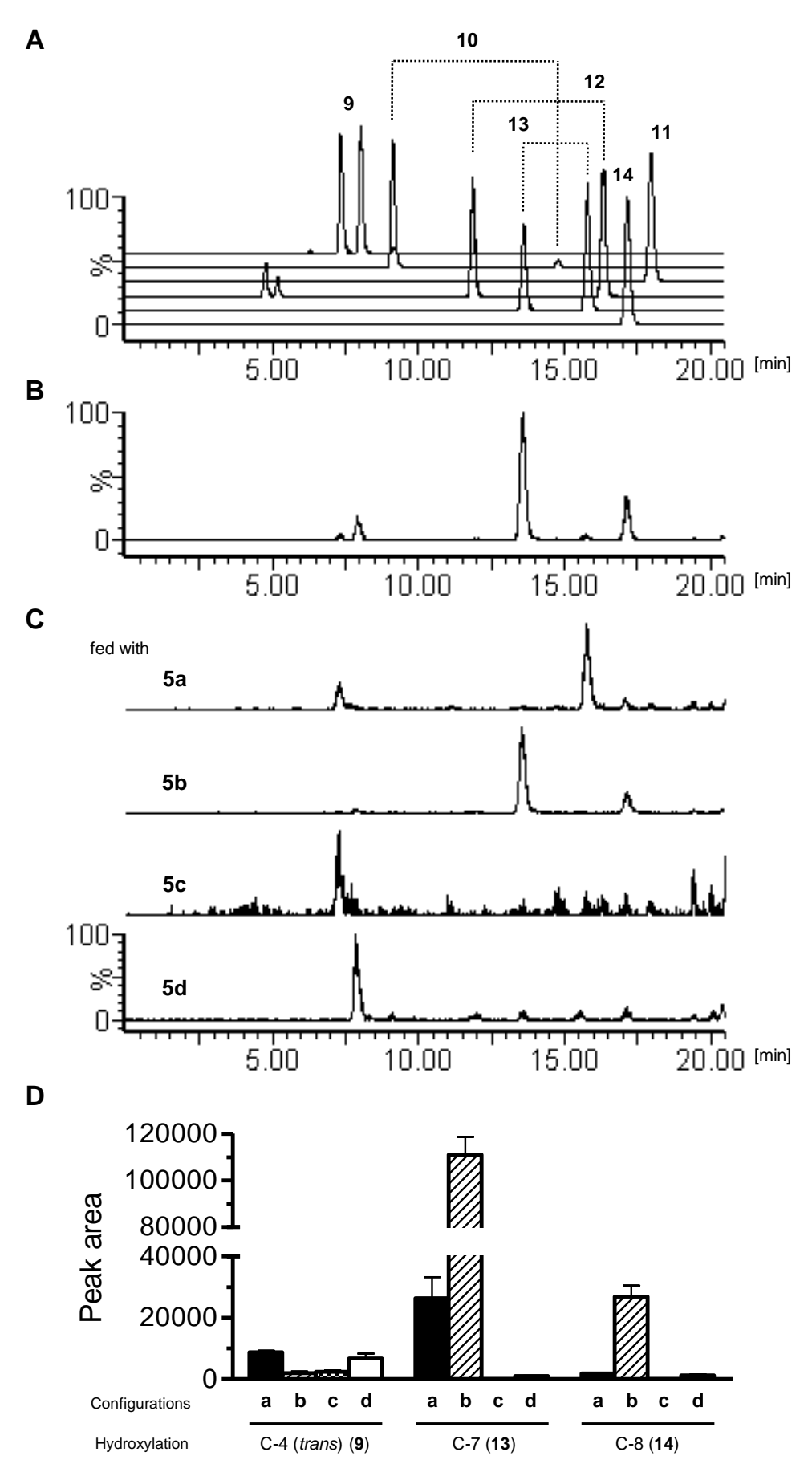


Fig. 6

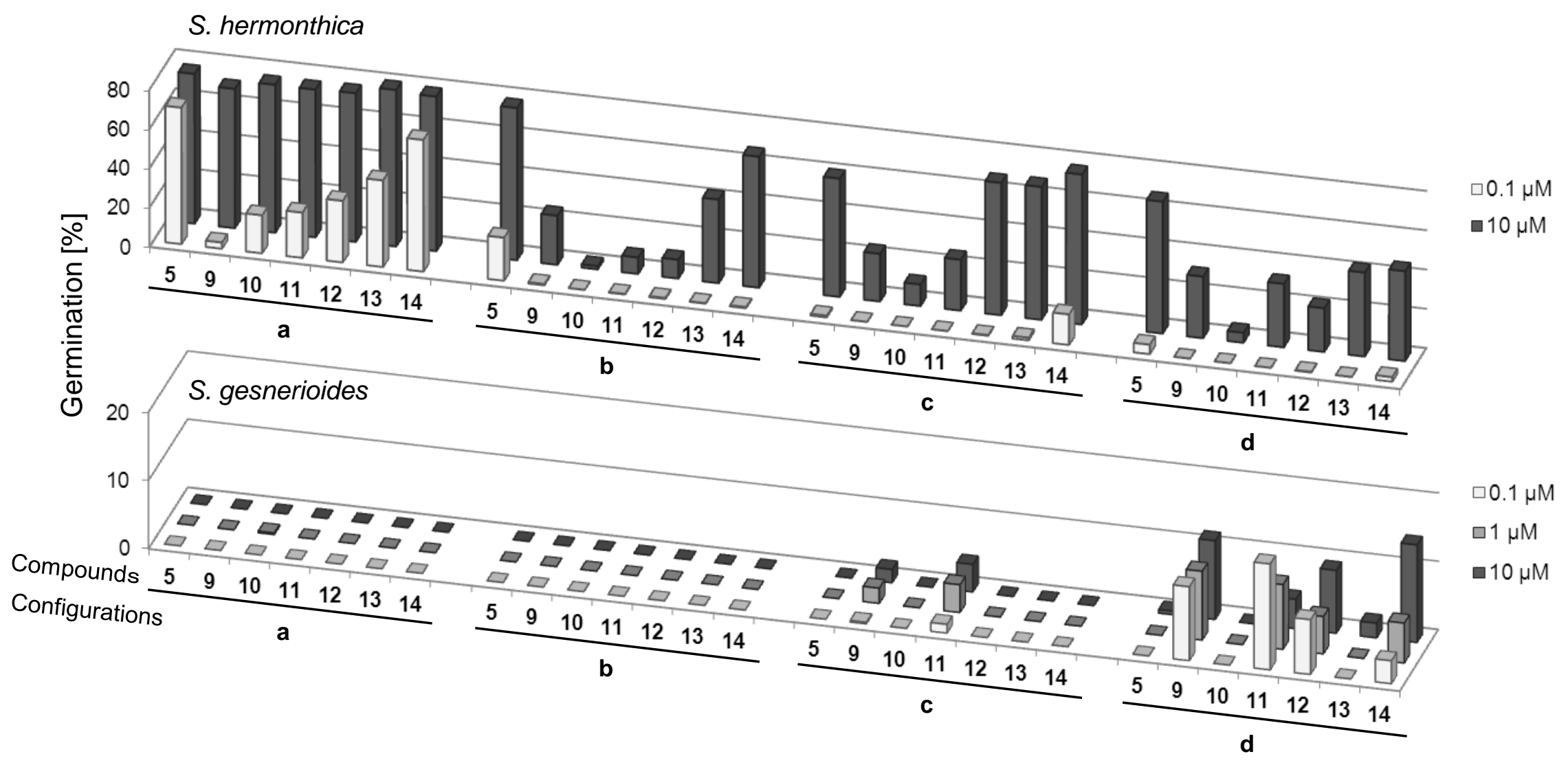



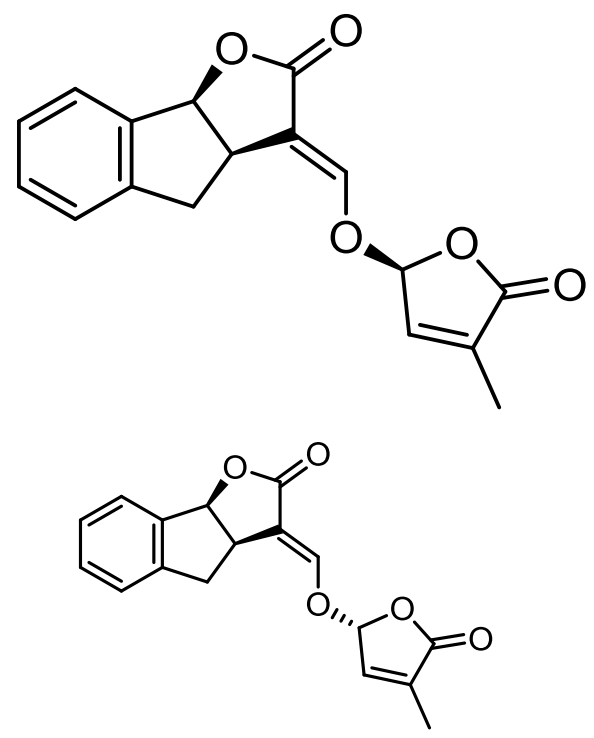

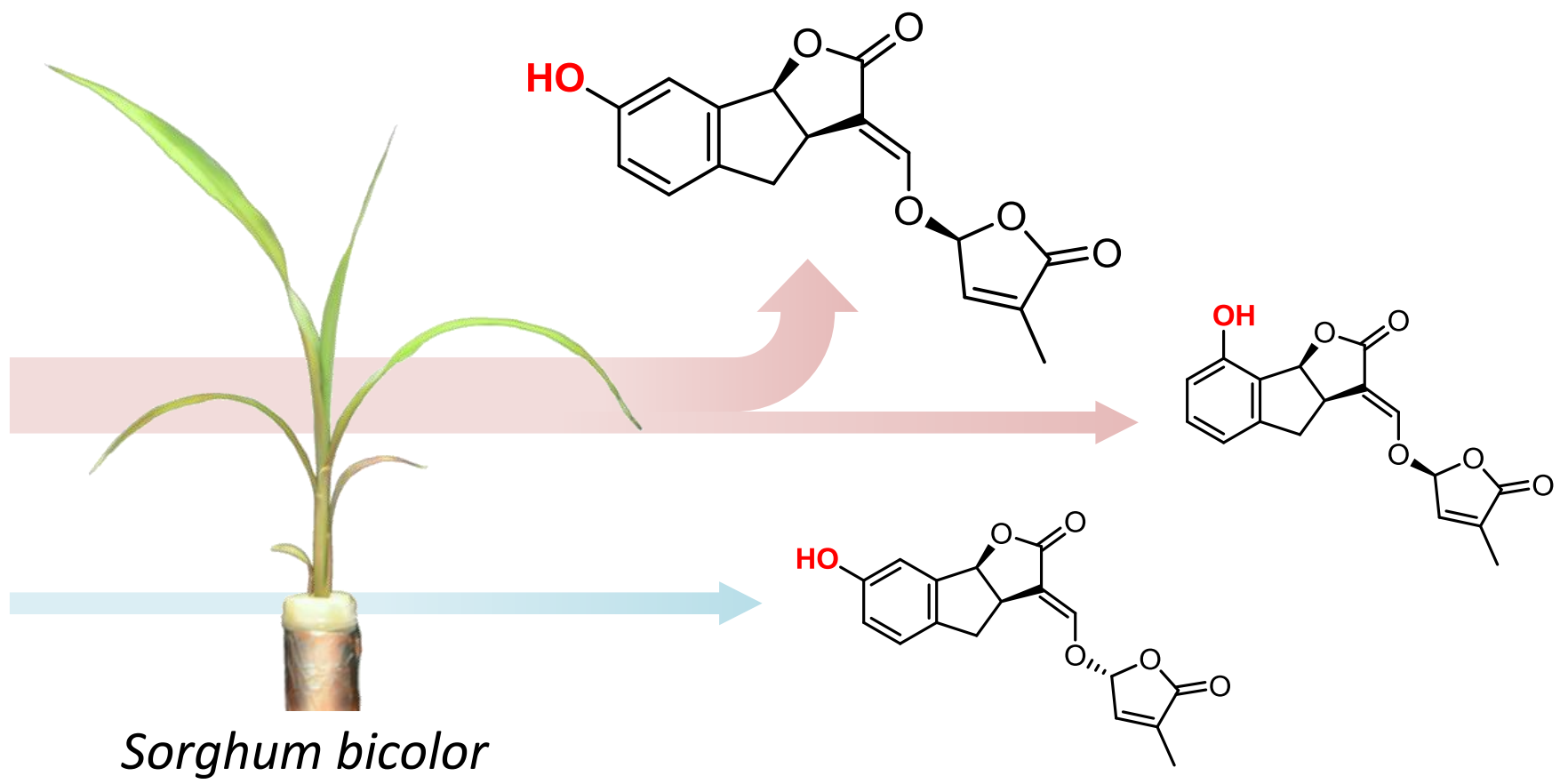

